\title{
Cycles and Multiple Equilibria in the Market for Durable Lemons
}

\author{
Maarten C. W. Janssen \\ (Erasmus University Rotterdam) \\ and \\ Vladimir Karamychev \\ (Tinbergen Institute Rotterdam)
}

January 2000

\begin{abstract}
We investigate the nature of market failure in a dynamic version of Akerlof (1970) where identical cohorts of a durable good enter the market over time. In the dynamic model, equilibria with qualitatively different properties emerge. Typically, in equilibria of the dynamic model, sellers with higher quality wait in order to sell and wait more than sellers of lower quality. Among other things, we show for any distribution of quality that there exist an infinite number of cyclical equilibria where all goods are traded within a certain number of periods after entering the market.
\end{abstract}

Key Words: Dynamic Trading, Asymmetric Information, Entry, Durable Goods.

JEL Classification: D82

Address for Correspondence: Tinbergen Institute Rotterdam, H16-18, Burg. Oudlaan 50, 3062 PA Rotterdam, The Netherlands; tel.: +31-(10)-4081298, fax: +31-(10)-4088900, E-mail: karamychev@FEW.EUR.NL.

We thank Hugo Hopenhayn and Santanu Roy for helpful suggestions. We have gained from comments made by members of the audience during presentations of this paper at the June 1999 conference on "Thirty Years after "The Market for Lemons" (Rotterdam), the 1999 European Meetings of the Econometric Society (Santiago). 


\section{Introduction}

Since the pioneering work of Akerlof (1970) many economists have studied the market failures due to asymmetric information in an otherwise perfectly competitive market. The standard model studies a static market with atomistic agents whose valuations depend on quality and a standard result is that only low quality goods are traded (if at all) even if the buyers are willing to pay more than the reservation price of sellers for each individual quality (see also, Wilson, 1979, 1980). This so-called lemons problem affects a large spectrum of markets, including insurance markets. In many cases, including the classic second-hand car market, the good under consideration is a durable good.

Durability introduces two complicating factors in the used goods markets: goods not traded in any period can be offered for sale in the future and, in addition, new cohorts of potential sellers may enter the market over time. Janssen and Roy (1999a, 1999b) have investigated some of the issues that arise when durability is explicitly taken into account in a dynamic model. Janssen and Roy (1999a) address the issue whether a given stock of goods can be traded over time. They show that in any dynamic competitive equilibrium all goods eventually will be traded. The main idea behind this result is that low quality sellers have less incentives to wait (before selling) compared to high quality sellers. Once certain (low) qualities are sold, only relatively high qualities remain in the market. Consumers can predict that sellers of different qualities will sort themselves into different time periods and, hence, they are willing to pay higher prices in later periods. The equilibrium is thus one in which higher qualities are sold in later periods at higher prices.

Janssen and Roy (1999b) address the same issue in the context of markets where identical cohorts of goods with uniformly distributed quality enter the market over time. In such markets, the infinite repetition of the static equilibrium under adverse selection is an equilibrium in the dynamic model. In fact, it is the unique stationary equilibrium and also the only equilibrium where prices and average quality traded are (weakly) monotonic over time. They show that there exists at least one other equilibrium, however, where all goods are traded within finite time after they have entered the 
market. These equilibria are cyclical in prices and quantities in the sense that once all goods are traded, prices (and quantities) will fall. Up to the moment all goods are sold, however, the dynamic process of prices and quantities is monotonically increasing.

In this paper we extend the analysis of Janssen and Roy (1999b) in a number of ways. First, we relax the assumption that in every period a cohort of uniformly distributed qualities enters the market. Instead, we allow for any arbitrary distribution, which satisfies some mild regularity condition. Second, our results are stronger in the sense that we show the existence of an infinite number of equilibria, where all goods are traded within finite time after they have entered the market. Finally, we show the extent to which the uniform distribution is special. It turns out that for a set of values of the model's parameters and a set of distributions, which have relatively little probability mass in the neighborhood of the static equilibrium, it is impossible to construct a dynamic equilibrium with monotonically increasing prices and quantities up to the moment everything is sold. We provide an example where this is the case. Hence, the equilibrium construction for the uniform distribution does not extend naturally to the class of all distributions.

Other existing literature ${ }^{1}$ on adverse selection has focused on various processes (such as signaling and screening) through which the difficulties of trading under asymmetric information may be resolved and has emphasized the role of non-market institutions in this context (such as certification intermediaries and leasing). This paper, in contrast, is motivated by a more basic issue which also underlies the original Akerlof paper viz., the functioning of the price mechanism in a perfectly competitive market when traders have private information. It is important to understand the nature of market failures due to adverse selection before analyzing the role of institutions in mitigating these failures.

Our specific model is as follows. We consider a competitive market for a perfectly durable good where potential sellers are privately informed about the quality of the goods they own. Each period, a cohort of sellers of equal size and with an identical, but arbitrary, distribution of quality enters the market. The demand side is 
modeled in the following simple way. Buyers are identical, have unit demand and for any given quality, a buyer's willingness to pay exceeds the reservation price of a seller for that quality. As buyers do not know the quality, their willingness to pay in a period equals the expected valuation of goods traded in that period. Moreover, there are more buyers than sellers in each period so that in equilibrium, prices equal the expected valuation. Once traded, goods are not re-sold in the same market. ${ }^{2}$

The Akerlof-Wilson model can be considered the static version of our model. The adverse selection problem implies that in equilibrium only a certain range of low qualities is traded. The infinitely repeated version of a static equilibrium outcome is also an equilibrium in our dynamic model. Hence, the issue of existence of dynamic equilibria is easily resolved. In this dynamic equilibrium high quality goods remain unsold forever.

We concentrate on the existence of other equilibria with more interesting properties - where prices and average quality traded fluctuate over time. We provide a characterization result saying that in all such equilibria the range of quality, which is eventually traded in the market, exceeds that in the stationary (static) outcome. Moreover, sellers of different qualities within each cohort of entrants separate themselves out over time. As the use value of low quality goods is lower than that of high quality goods, low quality sellers sell earlier than high quality sellers, the owner of a good with lower quality trades earlier, owners of higher quality goods wait longer. In order to highlight the waiting aspect of the adverse selection problem and also to make clear the sharp contrast between the properties of equilibria of our model with those of the static model, the main part of the analysis is devoted to proving the existence of an infinite number of equilibrium where every potential seller entering the market trades within a certain finite number of periods after entering the market.

The results obtained in the paper provide a different perspective on the adverse selection problem. In the static Akerlof-Wilson model, the adverse selection problem

1 See, for instance, Guha and Waldman (1997), Hendel and Lizzeri (1999a,b), Lizzeri (1999) and Waldman, (1999).

2 Our analysis bears some resemblance to that by Sobel (1991) of a durable goods monopoly where new cohorts of consumers enter the market over time. Unlike our framework, there is no correlation between the valuations of buyers and sellers in his model. 
manifests itself in the fact that relatively high quality goods cannot be traded despite the potential gains from trade. In the dynamic market for durable goods, the lemons problem is not so much the impossibility of trading relatively high quality goods, but rather that sellers with relatively high quality goods need to wait longer in order to trade. ${ }^{3}$ So, the cost of waiting becomes an important factor in the welfare loss arising due to asymmetric information.

There are three important intertemporal factors in the market which determine the market dynamics in all the non-stationary equilibria of our model. First, once a certain range of quality is traded, only sellers of higher quality goods are left in the market, which tends to improve the distribution of quality of potentially tradable goods in the future. Second, the entry of a new cohort of potential sellers with goods of all possible quality dilutes the average quality of potentially tradable goods - as they cannot be distinguished by buyers from higher quality sellers left over from the past. Finally, as time progresses and stocks of untraded goods accumulate from the past, the new cohort of traders entering the market in any period becomes increasingly less significant in determining the distribution of quality of tradable goods. ${ }^{4}$

The paper is organized as follows. Section 2 sets out the model, the equilibrium concept and some preliminary results. Section 3 provides a characterization result. The main result of the paper relating to the existence of an infinite number of equilibria where all goods are traded within finite time after entry into the market are outlined in section 4. Section 5 concludes. Proofs are contained in the Appendix.

\footnotetext{
3 There are certain situations in which the fact that a seller has waited for a long time might indicate low rather than high quality. This would be true, for example, when the buyers can inspect quality - high valuation buyers are more likely to inspect and select the relatively high quality houses - leaving unsold goods of relatively low quality for later periods (Taylor, 1998). A paper with a similar spirit is that of Vettas (1997). As stated earlier, our model is designed to understand the nature of the lemons problem and so we do not allow for any technology which can directly modify the information structure.

4 If there is no entry of sellers after the initial period, or equivalently, if buyers can distinguish the period of entry of sellers in the market, then only the first factor is relevant. In that case, it has been shown earlier for fairly general distributions of quality (see, Janssen and Roy (1998)) that in every equilibrium all goods are traded in finite time. Vincent (1990) analyzes a dynamic auction game with similar features.
} 


\section{Model.}

Consider a Walrasian market for a perfectly durable good whose quality, denoted by $\theta$, varies between $\underline{\theta}$ and $\bar{\theta}$, where $0<\underline{\theta}<\bar{\theta}<\infty$. Time is discrete and is indexed by $t=1,2, \ldots \infty$. Each time period $t$ a set of sellers $I_{t}$ enters the market, $I$ is the set of all sellers, $I=\bigcup_{t=1}^{\infty} I_{t}$ and $t_{i}$ is the period of entry of seller $i \in I$. Each seller is endowed with one unit of the durable good of quality $\theta_{i}$. Let the total Lebesgue measure of sellers from the set $I_{t}$ who own a good of quality less than or equal to $\theta$ be a function $\mu\left(\left\{i \mid i \in I_{t}, \theta_{i} \leq \theta\right\}\right) \equiv \mu(\theta)$, which is independent of $t$. We assume that $\mu(\theta)$ is strictly increasing and absolutely continuous with respect to the Lebesgue measure.

The measure of all sellers who enter the market in each period is strictly positive, so $\mu(\bar{\theta})>0$. Each seller $i$ knows the quality $\theta_{i}$ of the good he is endowed with and derives flow utility from ownership of the good until he sells it. Therefore, the seller's reservation price is the discounted sum of gross surplus due to ownership and we assume that it is exactly equal to $\theta_{i}$. So, the per period gross surplus is $(1-\delta) \theta_{i}$.

Each time period $t$ a set of buyers, with measure larger than $\mu(\bar{\theta})$, enters the market. All buyers are identical and have unit demand. A buyer's valuation of quality $\theta$ is equal to $v \theta$, where $v>1$. Thus, under full information, a buyer's valuation exceeds the seller's. All buyers know the ex ante distribution of the sellers with respect to qualities but do not know the quality of the good offered by a particular seller. When a buyer buys a good he leaves the market forever. All players discount the future with common discount factor $\delta, 0<\delta<1$. They are risk neutral and rational agents.

We will denote expected quality of the good from seller $i$ conditional on the fact that he belongs to a certain subset $I^{\prime} \subset I$ as $\eta\left(\left\{I^{\prime}\right\}\right)$, this value is defined for all $I^{\prime} \subset I$ such that $\mu\left(\left\{I^{\prime}\right\}\right)>0$ and it follows that

$$
\eta\left(I^{\prime}\right) \equiv \frac{1}{\mu\left(I^{\prime}\right)} \int_{i \in I^{\prime}} \theta_{i} d \mu\left(I^{\prime}\right)
$$


In order to have an adverse selection problem in the static model we assume $v E(\theta)<\bar{\theta}$, where $E(\theta)$ is the unconditional expected quality of all goods, $E(\theta)=\eta\left(\left\{I_{t}\right\}\right)=\eta(\{I\})$. This implies that the static Akerlof-Wilson version of this model has a largest equilibrium quality, which we will denote by $\theta_{S} \in(\underline{\theta}, \bar{\theta})$ :

$$
\theta_{S}=\max _{\theta}\left\{\theta \mid \nu \eta\left(\left\{i \mid i \in I, \theta_{i} \in[\underline{\theta}, \theta]\right\}\right)=\theta\right\} .
$$

To simplify our analysis we make the following two assumptions. Throughout this paper, we assume that these assumptions hold. Basically assumptions 2.1 and 2.2 assure that the distribution of quality is sufficiently well-behaved for some leftneighborhood of $\theta_{S}$.

Assumption 2.1. Let $\mu(\theta)$ be a strictly increasing and absolutely continuous with respect to Lebesgue measure on $\left[\theta_{S}-\varepsilon_{\mu}, \bar{\theta}\right]$ for some $\varepsilon_{\mu}>0$. Moreover let $\exists m_{\mu}, M_{\mu}$ such that for any $\theta^{\prime}, \theta^{\prime \prime}, \theta_{S}-\varepsilon_{\mu} \leq \theta^{\prime}<\theta^{\prime \prime} \leq \bar{\theta}: 0<m_{\mu}<\frac{\mu\left(\theta^{\prime \prime}\right)-\mu\left(\theta^{\prime}\right)}{\left(\theta^{\prime \prime}-\theta^{\prime}\right)}<M_{\mu}$.

Assumption 2.2. The measure function $\mu(\theta)$ is a differentiable function at $\theta=\theta_{S}$ and $\frac{d \mu(\theta)}{d \theta}\left(\theta_{s}\right)=f \geq m_{\mu}>0$

Given a sequence of market prices $\mathbf{p}=\left\{p_{t}\right\}_{t=1}^{\infty}$ each seller $i$ chooses whether or not to sell and if he chooses to sell, the time period in which to sell. If he chooses not to sell his gross surplus is equal to $\theta_{i}$ and therefore his net surplus equals to zero, while if he decides to sell in period $t \geq t_{i}$ his gross surplus is

$$
\sum_{\tau=t_{i}}^{t-1}(1-\delta) \theta_{i} \delta^{\tau-t_{i}}+\delta^{t-t_{i}} p_{t}=(1-\delta) \theta_{i} \frac{1-\delta^{t-t_{i}}}{(1-\delta)}+\delta^{t-t_{i}} p_{t}=\theta_{i}\left(1-\delta^{t-t_{i}}\right)+\delta^{t-t_{i}} p_{t}
$$

and, therefore, his net surplus equals

$$
s_{i}=\theta_{i}\left(1-\delta^{t-t_{i}}\right)+\delta^{t-t_{i}} p_{t}-\theta_{i}=\left(p_{t}-\theta_{i}\right) \delta^{t-t_{i}} .
$$

The set of time periods in which it is optimal to sell for a seller $i$ is given by

$$
T_{i}(\mathbf{p}) \equiv \underset{t \geq t_{i}}{\arg \max }\left\{s_{i} \mid s_{i} \geq 0\right\}=\underset{t \geq t_{i}}{\arg \max }\left\{\left(p_{t}-\theta_{i}\right) \delta^{t-t_{i}} \mid\left(p_{t}-\theta_{i}\right) \geq 0\right\} .
$$


If $p_{t}-\theta_{i}<0$ for all $t \geq t_{i}$ then $T_{i}(\mathbf{p})=\varnothing$.

Each potential seller $i$ chooses a time period $\tau_{i} \in T_{i}$ in which to sell. Let $\mathbf{t}=\left\{\tau_{i}\right\}_{i \in I}$ be a set of all selling decisions. We will denote a set of the sellers who chose time period $t$ for trade as $J_{t}$, and it follows that $J_{t} \equiv\left\{i \in I \mid \tau_{i}=t\right\}$. This generates a certain distribution of qualities over all time periods and the expected quality of the goods offered for sale in time period $t$ is $\eta_{t}=\eta\left(\left\{J_{t}\right\}\right)$ when $\mu\left(\left\{J_{t}\right\}\right)>0$.

In the sections that follow we will use the following notation:

a) $\mu\left(\theta_{\tau-1}, \theta_{\tau}\right)=\mu\left(\theta_{\tau}\right)-\mu\left(\theta_{\tau-1}\right)$, so that for all $\theta_{S}-\varepsilon_{\mu} \leq \theta_{\tau-1} \leq \theta_{\tau}<\infty \mu\left(\theta_{\tau-1}, \theta_{\tau}\right)$ is the measure of sellers from $I_{t}$ whose goods are of quality from the range $\left[\theta_{\tau-1}, \theta_{\tau}\right]: \mu\left(\theta_{\tau-1}, \theta_{\tau}\right)=\mu\left(\left\{i \mid i \in I_{t}, \theta_{i} \in\left[\theta_{\tau-1}, \theta_{\tau}\right]\right\}\right)$ and $\mu\left(\theta_{\tau}, \theta_{\tau-1}\right)=-\mu\left(\theta_{\tau-1}, \theta_{\tau}\right)$;

b) $\eta\left(\theta_{\tau-1}, \theta_{\tau}\right)=\left\{\begin{array}{r}\left.\frac{1}{\mu\left(\theta_{\tau-1}, \theta_{\tau}\right)}\right)_{\theta_{\tau-1}}^{\theta_{\tau}} \theta d \mu, \text { if } \theta_{\tau} \neq \theta_{\tau-1} \\ \theta_{\tau-1}, \text { if } \theta_{\tau}=\theta_{\tau-1}\end{array}\right.$

so that for all $\theta_{S}-\varepsilon_{\mu} \leq \theta_{\tau-1} \leq \theta_{\tau} \leq \bar{\theta}, \eta\left(\theta_{\tau-1}, \theta_{\tau}\right)$ is the expected quality of goods from sellers who belong to $I_{t}$ whose goods are of quality from the range $\left[\theta_{\tau-1}, \theta_{\tau}\right]$ : $\eta\left(\theta_{\tau-1}, \theta_{\tau}\right)=\eta\left(\left\{i \mid i \in I_{t}, \theta_{i} \in\left[\theta_{\tau-1}, \theta_{\tau}\right]\right\}\right)$, and $\eta\left(\theta_{\tau}, \theta_{\tau-1}\right)=\eta\left(\theta_{\tau-1}, \theta_{\tau}\right)$.

The following lemma states the continuity of $\eta\left(\theta_{\tau-1}, \theta_{\tau}\right)$.

Lemma 2.1. For all $\theta_{S}-\varepsilon_{\mu} \leq \theta_{\tau-1} \leq \theta_{\tau} \leq \bar{\theta}$ the function $\eta\left(\theta_{\tau-1}, \theta_{\tau}\right)$ is a strictly increasing continuous function. Moreover, $\exists m_{\eta}, M_{\eta}$, such that

$$
0<m_{\eta}<\frac{\eta\left(\theta_{\tau-1}, \theta_{\tau}\right)-\eta\left(\theta_{\tau-1}, \theta_{\tau-1}\right)}{\left(\theta_{\tau}-\theta_{\tau-1}\right)}<M_{\eta} .
$$

On the equilibrium path, buyers' expectations of quality in a period where a strictly positive measure of goods is offered for sale must equal the expected quality in that time period. As all buyers are identical, we assume that their expectations of quality in period $t$ are symmetric and denoted by $E_{t}$. 
A dynamic equilibrium is an equilibrium where all players rationally maximize their objectives, expectations are fulfilled and markets clear in every period.

Definition 2.1. A dynamic equilibrium is described in terms of: a sequence of prices $\mathbf{p}=\left\{p_{t}\right\}_{t=1}^{\infty}$, a set of selling decision $\mathbf{t}=\left\{\tau_{i}\right\}_{i \in I}$ and a sequence of buyers' quality expectations $\mathbf{E}=\left\{E_{t}\right\}_{t=1}^{\infty}$ such that:

a) Seller maximize: $\tau_{i} \in T_{i}(\mathbf{p})$ for all $i \in I$, i.e., seller $i$ chooses time period $\tau_{i}$ to trade optimally.

b) Buyers maximize and market clear: If $\mu\left(\left\{J_{t}\right\}\right)>0$ then $p_{t}=v E_{t}$, i.e., if there is a strictly positive amount of trade in time period $t$, then each buyer earns zero net surplus so that he is indifferent between buying and not buying and market clears. If $\mu\left(\left\{J_{t}\right\}\right)=0$ then $p_{t} \geq v E_{t}$, i.e., if zero measure of trade occurs in time period $t$ then each buyer can earn at most zero net surplus. Hence, not buying is optimal for him in that period.

c) Expectations are fulfilled when trade occurs: If $\mu\left(\left\{J_{t}\right\}\right)>0$ then $E_{t}=\eta_{t}$.

d) Expectations are reasonable even if no trade occurs: For all $t E_{t} \geq \underline{\theta}$.

Given the set-up described above, conditions (a)-(c) are quite standard, condition (d) says that even in periods in which (at most) zero measure of seller intends to sell, buyers' beliefs should be reasonable. This condition assures that autarky, i.e., no trade in any period, cannot be sustained in an equilibrium of the dynamic model. Given the condition, the willingness to pay, hence the price in any period, is restricted from below by $\underline{\imath}$ and sellers with low enough qualities prefer to sell against this price rather than not sell.

\section{Characterization of equilibrium.}

We start the analysis characterizing the properties of any dynamic equilibrium. In the Proposition 3.1 below we first argue that if a good of certain quality sells in period $t$, then all goods with lower qualities that have entered the market in and before period $t$ 
will also sell in that period. This fact allows us to define for each period a marginal seller $\theta_{t}$ as the seller of the highest quality in period $t$. It also allows us to define $s_{t}$ as the surplus of the marginal seller in period $t$, i.e., $s_{t}=p_{t}-\theta_{t}$. This part of the Proposition 3.1 basically follows from the fact that the use value of low qualities is lower than the use value of high qualities so that low qualities are more ready to sell.

The second part of the Proposition 3.1 argues that the marginal sellers in any period make non-negative net surplus. This implies that the other sellers make strictly positive surplus.

The third part of the Proposition 3.1 argues that the marginal seller in period $t$ is indifferent between selling in period $t$ and selling in the first future period in which a quality larger than his own quality is sold. Prices in that future period will be higher, reflecting higher average quality, but the discounted surplus is such that the seller is indifferent.

The last part of the Proposition 3.1 says that if it exists the highest quality that will ever be sold in any dynamic equilibrium is either equal to $\bar{\theta}$ or it is such that the seller makes zero surplus. It is clear that if a seller makes zero net surplus, prices in all future periods cannot be higher as this seller will have an incentive to wait and sell in that future period. The Proposition 3.1 argues that if the highest quality sold in a dynamic equilibrium makes strictly positive surplus, then it must be equal to $\bar{\theta}$.

Proposition 3.1 (equilibrium characterization). Any dynamic equilibrium has the following properties.

a) For all $t \exists \theta_{t} \in[\underline{\theta}, \bar{\theta}]$ such that if $\theta_{t}>\underline{\theta}$ then $J_{t}=\left\{i \theta_{i} \in\left[\underline{\theta}, \theta_{t}\right], t_{i} \leq t\right\}$, i.e., in every period $t$ in which strictly positive amount of trade occurs the set of quality traded is a range $\left[\underline{\theta}, \theta_{t}\right]$, where $\theta_{t}$ is the marginal quality traded in period $t$. All sellers who are in the market by period $t$ and own goods of quality not larger than $\theta_{t}$ prefer to trade in this time period.

b) $p_{t} \geq \theta_{t}$. 
c) Let $\tilde{t}(t)=\min _{\tau>t}\left\{\tau \mid \theta_{\tau}>\theta_{t}\right\}$, i.e., $\tilde{t}(t)$ is the first period after $t$ where $\theta_{\tau}>\theta_{t}$. Then $p_{t}-\theta_{t}=\delta^{\tilde{f}(t)-t}\left(p_{\tilde{t}(t)}-\theta_{t}\right)$, i.e., marginal seller in period $t$ is just indifferent between selling in that period and in the first next period where marginal quality is larger than his own quality.

d) If $\hat{t}(t)=\min \left(\underset{\tau>t}{\arg \max } \theta_{\tau}\right)$ then $\left(p_{\hat{t}(t)}-\theta_{\hat{t}(t)}\right)\left(\bar{\theta}-\theta_{\hat{t}(t)}\right)=0$, i.e., if in period $\hat{t}$ the marginal quality is the first largest one for all subsequent periods then either this is the highest possible quality $\bar{\theta}$ or the surplus of the corresponding marginal seller is zero.

It is easily seen that the infinitely repeated outcome of the static model is a dynamic equilibrium of our model. Hence, existence of equilibrium is not really an issue here. We will show that in the dynamic model there are infinitely many other equilibria, each one starting from a certain neighborhood of a static equilibrium quality.

\section{Equilibria Trading all Goods.}

We will now show that for any measure function $\mu(\theta)$ which satisfies Assumptions 2.1-2.2 and for all generic values of the parameters $v, \delta, \underline{\theta}$ and $\bar{\theta}$ there exist an infinite number of dynamic equilibria covering all qualities up to $\bar{\theta}$. As we already know that our model has at least one equilibrium, a general existence proof is trivial. That is why we use a constructive proof showing how to find an equilibrium sequence of marginal qualities that is such that all qualities up to $\bar{\theta}$ are traded. The fact that there are infinitely many dynamic equilibria follows from the fact that if there exists a dynamic equilibrium covering all qualities up to $\bar{\theta}$ starting from some $\theta_{1}<\theta_{S}$, then there also exists a dynamic equilibrium covering all qualities up to $\bar{\theta}$ starting from a $\theta_{1}^{\prime}$, with $\theta_{1}<\theta_{1}^{\prime}<\theta_{S}$. 
Before we will go into the details of the analysis, we first introduce an important parameter. Assumption 2.2 allows us to define a parameter $a$, which describes the relation between the distribution of quality over the range $\left[\underline{\theta}, \theta_{S}\right]$ and the marginal distribution at $\theta_{S}$ itself:

$$
a \equiv \frac{1}{\mu\left(\theta_{S}\right)}(v-1) \theta_{S} \frac{d \mu(\theta)}{d \theta}\left(\theta_{s}\right)=\frac{(v-1) \theta_{S} f}{\mu\left(\theta_{S}\right)} .
$$

Obviously, $a$ is strictly positive. We will now argue that generically, it must be that $a<1$. To this end, consider the surplus of the marginal seller in the static model as a function of $\theta$ :

$$
\begin{aligned}
& s(\theta) \equiv p(\theta)-\theta=v \eta(\theta)-\theta=v \frac{1}{\mu(\theta)} \int_{\underline{\theta}}^{\theta} \theta d \mu(\theta)-\theta, \text { and } \\
& \frac{d s(\theta)}{d \theta}\left(\theta_{S}\right)=v \frac{d}{d \theta}\left(\frac{1}{\mu(\theta)} \int_{\underline{\theta}}^{\theta} \theta d \mu(\theta)\right)\left(\theta_{S}\right)-1=a-1 .
\end{aligned}
$$

Thus, the surplus of the marginal seller can be written as

$$
s(\theta) \equiv s\left(\theta_{S}\right)+\frac{d s(\theta)}{d \theta}\left(\theta_{S}\right)\left(\theta-\theta_{S}\right)+o\left(\theta-\theta_{S}\right)=(a-1)\left(\theta-\theta_{S}\right)+o\left(\theta-\theta_{S}\right) .
$$

Suppose then that $a>1 .^{5}$ This would imply that $s(\theta)>0$ in some right neighborhood of $\theta_{S}$. But this contradicts the assumption that $\theta_{S}$ is the highest static equilibrium quality.

In the uniform case, we have $a=\frac{1}{2} v^{6}$. As in the uniform case adverse selection implies that $1<v<2$, the uniform distribution is a special case of the case when $a \in\left(\frac{1}{2}, 1\right)$. In subsection 4.1 we will start with this simplest case, which generalizes the analysis in Janssen and Roy (1999b). We show that one can construct a "monotonic" sequence of marginal qualities $\theta_{t}$ that are strictly increasing over time until all goods are sold. The main reason why the case $a \in\left(\frac{1}{2}, 1\right)$ is to be distinguished from other cases can be seen by looking at equation (1). If we choose $\theta_{1}=\theta_{S}$, then in the second period, the measure of qualities just above $\theta_{S}$ that are not yet sold is two times as high

\footnotetext{
5 The case where $a=1$ is a non-generic case.
} 
as the original measure. If $a \in\left(\frac{1}{2}, 1\right)$, the distribution of qualities in the second period is such that a new "static" equilibrium emerges that is larger than $\theta_{S}$. As in the second period we can write $s_{2}(\theta)=(2 a-1)\left(\theta_{2}-\theta_{S}\right)-a\left(\theta_{1}-\theta_{S}\right)+o\left(\left(\theta_{2}-\theta_{S}\right),\left(\theta_{1}-\theta_{S}\right)\right)$ it possible to choose $\theta_{1}$ close enough to $\theta_{S}$ such that $\theta_{2}>\theta_{1}$ and $s_{2}>0$.

If $a<\frac{1}{2}$, it may not be possible to construct such a "monotonic" equilibrium and we show this by example. In subsection 4.2 we show that dynamic equilibria nevertheless exist if $a \in(0, \tilde{a}(\delta))$, where $\tilde{a}(\delta)$ is some decreasing function of $\delta$. The kind of equilibrium we obtain is not monotonic, however, and marginal qualities $\theta_{t}$ strictly decrease for some initial time periods after which they strictly increase until all goods are sold. The general theorem is provided in subsection 4.3. As the construction here becomes quite complicated, subsections 4.1 and 4.2 are also provided for didactical reasons.

The construction of equilibrium uses an "equilibrium sequence" which is defined below.

Definition 4.1. An equilibrium sequence $\Theta_{T}(U)$ is a finite sequence of marginal qualities $\theta_{t}$ as functions of $\theta_{1}$, the latter being defined over some range $U=\left(\underline{\theta_{1}}, \overline{\theta_{1}}\right)$, i.e., $\Theta_{T}(U)=\left\{\theta_{t}\left(\theta_{1}\right)\right\}_{t=1}^{T}$, such that all equilibrium conditions in Definition 2.1 hold for all $t=1, \ldots, T-1$. Moreover, for all $\theta_{1} \in U$ :

a) $\theta_{t}\left(\theta_{1}\right)$ is continuous for all $t=1, \ldots, T$;

b) $\theta_{T}\left(\theta_{1}\right)>\theta_{t}\left(\theta_{1}\right)$ for all $t=1, \ldots, T-1$;

c) $\quad p_{t}=\nu \eta_{t}=p_{t}\left(\theta_{1}\right)$, the price in period $t$ is continuous and $p_{t}\left(\theta_{S}\right)=\theta_{S}$;

d) $p_{t}\left(\theta_{1}\right)>\theta_{t}\left(\theta_{1}\right)$ for all $t=1, \ldots, T$, i.e., the price in period $t$ exceeds the marginal quality in that period and $s_{t}\left(\theta_{1}\right)>0$;

\footnotetext{
${ }^{6}$ This easily follows from the fact that $\theta_{S}=\frac{v}{2-\nu} \underline{\theta}$ and $\mu\left(\theta_{S}\right)=f\left(\theta_{S}-\underline{\theta}\right)$.
} 
e) for all $t=1, \ldots, T-1 \quad p_{t}-\theta_{t}=\delta^{(\tilde{t}(t)-t)}\left(p_{\tilde{t}(t)}-\theta_{t}\right)$, where $\tilde{t}(t)=\min _{t<\tau \leq T}\left\{\tau \mid \theta_{\tau}>\theta_{t}\right\}$, i.e., the marginal seller in period $t$ is just indifferent between selling in that period and in the first next period where marginal quality is larger than his own quality. ${ }^{7}$

The above definition does not imply the existence of an equilibrium sequence. However, it easy to see that there exists at least one equilibrium sequence, namely $\Theta_{1}\left(\left(\theta_{S}-\varepsilon_{\mu}, \theta_{S}\right)\right)=\left\{\theta_{1}\right\}$, such that all mentioned above conditions are trivially satisfied.

The main property of an equilibrium sequence we use is that if there is a dynamic equilibrium with marginal qualities $\left\{\theta_{\tau}\right\}_{\tau=1}^{\infty}$ such that for $\tau=1, \ldots, T$ it can be described by a certain equilibrium sequence $\Theta_{T}(U)=\left\{\theta_{t}\left(\theta_{1}\right)\right\}_{t=1}^{T}$, then there is only one indifference equation, namely

$$
p_{T}-\theta_{T}=\delta^{(\tilde{t}(T)-T)}\left(p_{\tilde{t}(T)}-\theta_{T}\right),
$$

which relates prices $p_{\tau}$ and marginal qualities $\theta_{\tau}$ for $\tau=T+1, \ldots \infty$ to prices $p_{\tau}$ and marginal qualities $\theta_{\tau}$ for $\tau=1, \ldots, T$ (this follows from (b) above). Intuitively, $\theta_{T}$ summarizes all the relevant properties of the sequence of marginal qualities up to time period $T$. Our purpose, therefore, is to find an equilibrium sequence such that $\theta_{T}\left(\theta_{1}\right)=\bar{\theta}$ for some $T$ and $\theta_{1}$.

\subsection{The case where $a>\frac{1}{2}$.}

In this subsection we prove the existence of an increasing sequence $\left\{\theta_{t}\right\}_{t=1}^{T}$, where $\theta_{T}=\bar{\theta}$ when $a>\frac{1}{2}$. As the uniform distribution is a special case, the result obtained in this section show to what extent the results obtained in Janssen and Roy (1999b) can be generalized to allow for other types of distribution functions. The following theorem contains a statement of the formal result.

\footnotetext{
7 That minimum always exists as at least one $\theta_{\tau}$ is above $\theta_{t}$, namely $\theta_{T}>\theta_{t}$.
} 
Theorem 4.1. For any $a \in\left(\frac{1}{2}, 1\right)$ and for any generic value of $\bar{\theta}$, there exist an infinite number of dynamic equilibria such that all goods are sold in finite time after entering the market.

The proof consists of three steps. In Proposition 4.1 we prove that it is possible to construct an equilibrium sequence of an arbitrary length where marginal qualities $\left\{\theta_{t}\right\}$ are strictly increasing and very close to the static equilibrium quality $\theta_{S}$. Under these circumstances the main indifference equation (2) takes the following form:

$$
p_{t}-\theta_{t}=\delta\left(p_{t+1}-\theta_{t}\right) \text {. }
$$

In other words, the marginal seller in period $t$ is just indifferent between selling in that period and in the next period. We will denote such monotonic equilibrium sequences as $\Theta^{1}(U)$ and call a dynamic equilibrium, which is based on them, as "dynamic equilibrium of type I".

Proposition 4.1. If $a \in\left(\frac{1}{2}, 1\right)$, then there exist an infinite number of $\Theta^{1}(U)$. Moreover, $\exists \varepsilon_{\gamma}, \exists T_{0}$ such that for all $t>T_{0} \exists U_{t}^{0}=\left(\theta_{1}^{0}(t), \theta_{S}\right)$ and $\exists \Theta_{t}^{1}\left(U_{t}^{0}\right)$ such that:

a) for all $\tau=1, \ldots, t \theta_{\tau}\left(\theta_{1}\right)$ is differentiable at $\theta_{1}=\theta_{S}$ and $\theta_{\tau}\left(\theta_{S}\right)=\theta_{S}$;

b) for all $\theta_{1} \in U_{t}^{0} \quad 0<\theta_{t}\left(\theta_{1}\right)-\theta_{S}<\varepsilon_{\gamma} \frac{1}{\delta} s_{t}\left(\theta_{1}\right)$.

Proposition 4.1 implies that if $a \in\left(\frac{1}{2}, 1\right)$, we can construct an equilibrium sequence of an arbitrarily long length $t$ such that in period $t+1$ there will be more sellers with high quality $\left(\theta_{i}>\theta_{S}\right)$ goods than the number of sellers with low quality $\left(\theta_{i}<\theta_{S}\right)$. This allows us to expand the equilibrium sequence $\Theta_{t}$ for some more periods.

Next, in Proposition 4.2, we prove that when we are able to construct an equilibrium sequence of an arbitrary length where all marginal qualities belong to a certain neighborhood of $\theta_{S}$, then we can expand it in such a way that the surplus of the last marginal quality $\theta_{t}$ could be made any value between 0 and $(v-1) \theta_{t}$. More precisely, given any equilibrium sequence $\Theta_{t}$ with $0<\theta_{t}\left(\theta_{1}\right)-\theta_{S}<\varepsilon_{\gamma} \frac{1}{\delta} s_{t}\left(\theta_{1}\right)$ we can construct another sequence $\Theta_{t^{\prime}}$, where $t^{\prime}>t$, such that $\Theta_{t} \subset \Theta_{t^{\prime}}$ and $p_{t}\left(\theta_{1}\right)$ covers the 
whole interval $\left(\theta_{t}\left(\theta_{1}\right), v \theta_{t}\left(\theta_{1}\right)\right)$. The conditions under which the Proposition 4.2 holds are the same as the conclusion reached in Proposition 4.1. This is done as in later subsections we will also make use of it.

Proposition 4.2. If there exist $\varepsilon_{\gamma}>0$ and $T_{0}$ such that for all $t>T_{0} \exists U_{t}^{0}=\left(\theta_{1}^{0}(t), \theta_{S}\right)$ and $\exists \Theta_{t}\left(U_{t}^{0}\right)$ such that for all $\theta_{1} \in U_{t}^{0} \theta_{t}\left(\theta_{1}\right)-\theta_{S}<\varepsilon_{\gamma} \frac{1}{\delta} s_{t}\left(\theta_{1}\right)$, then for any $\varepsilon_{S}>0$ and $\varepsilon_{\theta}>0 \exists T_{S}$ such that for all $t \geq T_{S} \exists U_{t}^{S}=\left(\theta_{1}^{S}(t), \theta_{S}\right) \subset U_{t}^{0}$ and $\exists \Theta_{t}\left(U_{t}^{S}\right)$ such that:

a) for any $\theta_{1} \in U_{t}^{S}\left|\theta_{t}\left(\theta_{1}\right)-\theta_{S}\right|<\varepsilon_{\theta}$;

b) $s_{t}\left(\theta_{S}\right)=0$;

c) $s_{t}\left(\theta_{1}^{S}\right)>(v-1) \theta_{t}\left(\theta_{1}^{S}\right)-\varepsilon_{S}$.

Proposition 4.2 tells us that if we could trade goods for many time periods and, therefore, accumulate "high quality sellers", then we can organize trade in such a way that in the last time period of the equilibrium sequence "almost" all sellers who prefer to sell in that period will have goods of quality very close to $\theta_{S}$.

Finally, in Proposition 4.3 we prove that if we are able to trade goods along an equilibrium path from a certain range of qualities such that the price in the last period of the equilibrium sequence can be made any value between the marginal quality and buyer's valuations of the marginal quality, then we can expand that equilibrium sequence in such a way that wider range of qualities could be traded with the same properties. Doing so, after a finite number of iterations we generically can construct an equilibrium sequence where $\theta_{t}=\bar{\theta}$, i.e., all goods are traded by the period $t$.

Proposition 4.3. If $\exists \theta^{(k)} \in\left[\theta_{S}, \bar{\theta}\right)$ such that for any $\varepsilon_{S}>0$ and $\varepsilon_{\theta}>0 \quad \exists T_{S}^{(k)}$ such that for all $t>T_{S}^{(k)} \exists U_{t}^{(k)}=\left(\underline{\theta}_{1}(t, k), \bar{\theta}_{1}(t, k)\right)^{8}$ and $\exists \Theta_{t}\left(U_{t}^{(k)}\right)$ such that:

a) for all $\theta_{1} \in U_{t}^{(k)}\left|\theta_{t}\left(\theta_{1}\right)-\theta^{(k)}\right|<\varepsilon_{\theta}$;

\footnotetext{
${ }^{8}$ Here we don't make a distinction between $\underline{\theta}_{1}<\bar{\theta}_{1}$ and $\underline{\theta}_{1}>\bar{\theta}_{1}$. All we need is $U_{t}^{(k)}$ to be a nonempty open set while $\underline{\theta}_{1}$ and $\bar{\theta}_{1}$ are its boundary points.
} 
b) $0 \leq s_{t}\left(\underline{\theta}_{1}(t, k)\right)<\varepsilon_{S}$;

c) $s_{t}\left(\bar{\theta}_{1}(t, k)\right)>(v-1) \theta_{t}\left(\bar{\theta}_{1}(t, k)\right)-\varepsilon_{S}$;

then either

a) $\exists \overline{\varepsilon_{S}}>0$ such that for any $T \exists \bar{t}>T, \exists \tilde{\theta_{1}}(\bar{t})$ and $\exists \Theta_{\bar{t}}\left(\tilde{\theta_{1}}\right)$ such that $\theta_{\bar{t}}\left(\tilde{\theta_{1}}\right)=\bar{\theta}$ and $s_{\bar{t}}\left(\tilde{\theta_{1}}\right)>\overline{\varepsilon_{S}}$, or

b) for any $\overline{\varepsilon_{S}}>0$ and $\overline{\varepsilon_{\theta}}>0 \exists \hat{\theta}^{(k+1)} \in\left(\nu \hat{\theta}^{(k)}, \bar{\theta}\right]$ and $\exists T_{S}^{(k+1)}$ such that for all $t>T_{S}^{(k+1)}$ $\exists U_{t}^{(k+1)}=\left[\underline{\theta}_{1}(t, k+1), \bar{\theta}_{1}(t, k+1)\right] \subset U_{t}^{(k)}$ and $\exists \Theta_{t}\left(U_{t}^{(k+1)}\right)$ such that:

- for all $\theta_{1} \in U_{t}^{(k+1)} \theta_{t}\left(\theta_{1}\right)-\theta^{(k+1)} \mid<\overline{\varepsilon_{\theta}}$;

- $\quad 0 \leq s_{t}\left(\underline{\theta}_{1}(t, k+1)\right)<\overline{\varepsilon_{S}}$;

- $s_{t}\left(\bar{\theta}_{1}(t, k+1)\right)>(v-1) \theta_{t}\left(\bar{\theta}_{1}(t, k+1)\right)-\overline{\varepsilon_{S}}$.

Proposition 4.3 basically says that if we have constructed an equilibrium sequence for a sufficiently large number of periods, then we can either make sure that after some more time periods the next marginal quality can be chosen relatively far from the present marginal quality and such that all desirable properties are kept (case (b)) or we can reach $\bar{\theta}$ (case (a)).

Propositions 4.1, 4.2 and 4.3 taken together give us a large part of the proof of Theorem 4.1.

\subsection{The case of small $a$ and $\delta$.}

In this section, we construct an equilibrium sequence for the case when $a$ and $\delta$ small. We first provide an example showing why the analysis of the previous subsection does not continue to be valid. The example shows that when $a$ and $\delta$ are small there does not exist a $\theta_{2}>\theta_{1}$ such that $s_{2} \geq 0$ and such that $\theta_{1}$ is indifferent between selling in period 1 and selling in period 2. 
Example 4.1 Let us take $v=1.2, \delta=0.1, \underline{\theta}=10, \bar{\theta}=13$ and a measure function $\mu(\theta)$ such that $\mu(10)=0$ and

$$
\frac{d}{d \theta} \mu(\theta)= \begin{cases}101, & \text { if } \underline{\theta}<\theta<10.1 \\ 1, & \text { if } 10,1<\theta<\bar{\theta}\end{cases}
$$

The static equilibrium quality for this case is unique and equals $\theta_{S}=\sqrt{151.5} \approx 12.31$.

In any dynamic equilibrium we must have $\theta_{1} \in\left[\underline{\theta}, \theta_{S}\right]$ (otherwise we would have $\left.s_{1}<0\right)$. The following picture 4.1 shows the graph of functions $X 2=\theta_{2}(X 1)$ and $S 2=s_{2}(X 1)$ where $X 1=\theta_{1}$.

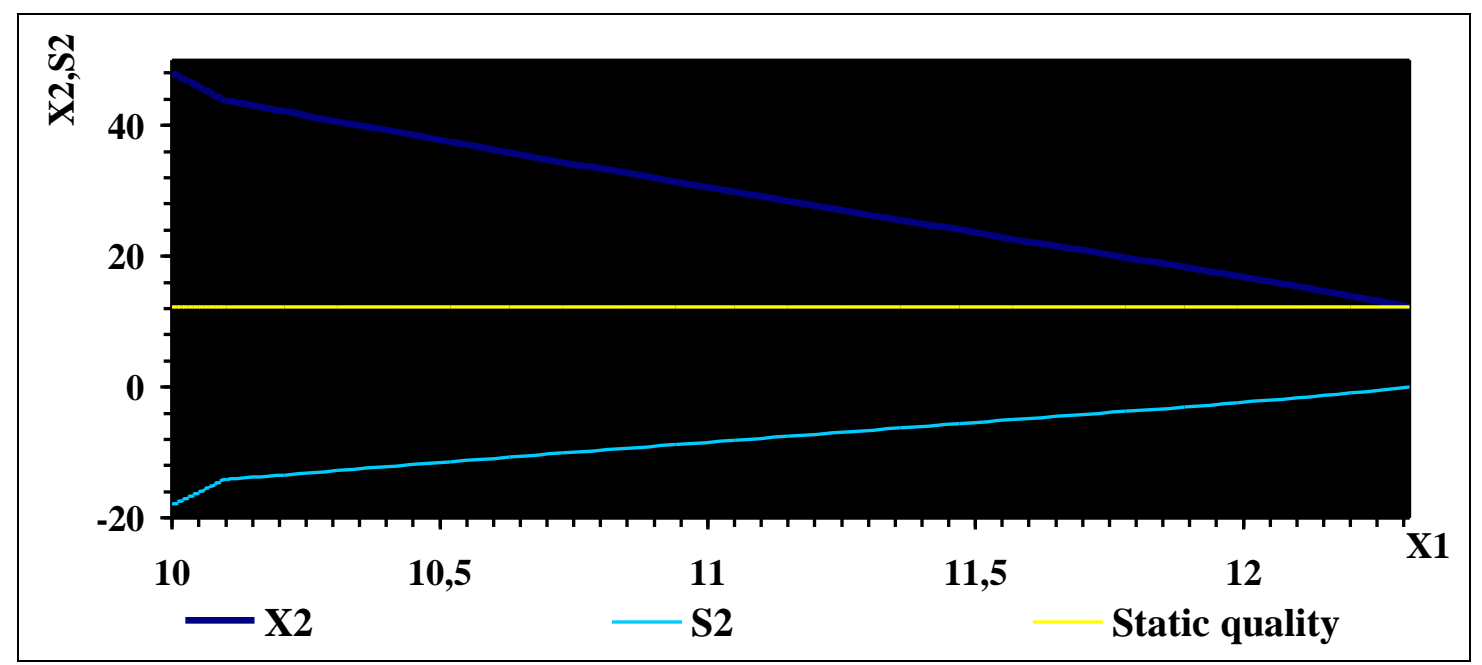

Figure 4.1.

It is easy to see that for any value of $\theta_{1}$ we get $\theta_{2}\left(\theta_{1}\right)$ above $\theta_{S}$ and the surplus in the second period is negative.

We will now prove that if $a$ is relatively small, particularly if $a \in\left(0,(1-\delta)^{2}\right)$, then we are still able to construct infinitely many dynamic equilibria such that all goods from the range $[\underline{\theta}, \bar{\theta}]$ are traded. The equilibrium sequence is non-monotonic. Note that the parameter configuration analyzed here partially overlaps with the parameter configuration analyzed in the previous subsection. The result we will prove is formally stated in Theorem 4.2 below. 
Theorem 4.2. For any $a \in\left(0,(1-\delta)^{2}\right)$, and for any generic value of $\bar{\theta}$, there exist an infinite number of dynamic equilibria such that all goods are sold in finite time after entering the market.

In order to prove this theorem we only need to show that when $a \in\left(0,(1-\delta)^{2}\right)$ it is also possible to construct an equilibrium sequence of an arbitrary large length $t$ where marginal qualities $\left\{\theta_{t}\right\}$ are very close to the static equilibrium quality $\theta_{S}$. We will construct a sequence that is strictly decreasing for some time, $\theta_{\tau+1}<\theta_{\tau}$, and only the last marginal quality $\theta_{T}$ exceeds all previous ones. We denote such a sequence as "equilibrium sequences of type II" and write $\Theta^{2}(U)$.

In this case our indifference equation (2) becomes the following system:

$$
p_{\tau}-\theta_{\tau}=\delta^{t-\tau}\left(p_{t}-\theta_{\tau}\right), \tau=1, \ldots, t
$$

Proposition 4.4. If $a \in\left(0,(1-\delta)^{2}\right)$, then there exist an infinite number of $\Theta^{2}(U)$. Moreover, $\exists \varepsilon_{\gamma}, \exists T_{0}$ such that for all $t>T_{0} \exists U_{t}^{0}=\left(\theta_{1}^{0}(t), \theta_{S}\right)$ and $\exists \Theta^{2}{ }_{t}\left(U_{t}^{0}\right)$ such that:

a) for all $\tau=1, \ldots, t \theta_{\tau}\left(\theta_{1}\right)$ is differentiable at $\theta_{1}=\theta_{S}$ and $\theta_{\tau}\left(\theta_{S}\right)=\theta_{S}$;

b) for all $\theta_{1} \in U_{t}^{0} \quad 0<\theta_{t}\left(\theta_{1}\right)-\theta_{S}<\varepsilon_{\gamma} \frac{1}{\delta} s_{t}\left(\theta_{1}\right)$.

Note that the conclusions reached in Proposition 4.4 are identical to the conclusions reached in Proposition 4.1 so that we can make use of Propositions 4.2 and 4.3 to get the proof of Theorem 4.2.

\subsection{The General case.}

Finally, we prove that for any value of $a$, we are able to construct infinitely many dynamic equilibria such that all goods in $[\underline{\theta}, \bar{\theta}]$ are traded. The structure of the corresponding equilibrium sequences becomes a mixture of the equilibrium sequences of type I and type II. 
Theorem 4.3. For any generic value of $\bar{\theta}$, there exist an infinite number of dynamic equilibria such that all goods are sold in finite time after entering the market.

Again, like in subsection 4.2, the only thing we need to prove is that it is possible to construct an equilibrium sequence of an arbitrary large length where marginal qualities $\left\{\theta_{t}\right\}$ are very close to the static equilibrium quality $\theta_{S}$. This is the content of Proposition 4.5.

Proposition 4.5. There exist an infinite number of $\Theta(U)$. Moreover, there exist some numbers $\varepsilon_{\gamma}, T_{0}$ and $k_{m} \geq 1$ such that for all $t>T_{0} \exists U_{t k_{m}+1}^{0}=\left(\theta_{1}^{0}\left(t k_{m}+1\right), \theta_{S}\right)$ and $\exists \Theta_{t k_{m}+1}\left(U_{t k_{m}+1}^{0}\right)$ such that:

a) for all $\tau=1, \ldots, t k_{m}+1 \theta_{\tau}\left(\theta_{1}\right)$ is differentiable at $\theta_{1}=\theta_{S}$ and $\theta_{\tau}\left(\theta_{S}\right)=\theta_{S}$;

b) for all $\theta_{1} \in U_{t k_{m}+1}^{0} 0<\theta_{t k_{m}+1}\left(\theta_{1}\right)-\theta_{S}<\varepsilon_{\gamma} \frac{1}{\delta} s_{t k_{m}+1}\left(\theta_{1}\right)$.

The main difference with related Propositions 4.1 and 4.4 is that here the equilibrium sequence constructed around $\theta_{S}$ is partly composed of increasing subsequences and partly composed of decreasing subsequences. Therefore, we need to indices $\left(t\right.$ and $\left.k_{m}\right)$ to keep track of the whole equilibrium sequence.

Note that the conclusions reached in Proposition 4.5 are identical to the conclusions reached in Proposition 4.1 so that we can make use of Propositions 4.2 and 4.3 to get the proof of Theorem 4.3.

\section{Conclusions}

In this paper, we have provided a different perspective on the way the adverse selection problem may manifest itself in durable good markets, where entry takes place in the same market. In the static Akerlof-Wilson model, adverse selection results in high quality goods not being able to trade despite the potential gains from trade. The infinite repetition of this static equilibrium is also an equilibrium in the dynamic model where a durable good is traded in a competitive market. Our main result in this paper, 
however, says that there are infinitely many other equilibria where all goods are sold within finite time after entering the market. In each of these dynamic equilibria, the marginal quality that is sold in the first period lies in a small neighborhood of the static equilibrium. This result holds true for all generic values of the parameters governing the behavior of buyers and sellers and the distribution of qualities in the population of sellers.

\section{References}

Akerlof, G., 1970, "The market for lemons: Qualitative uncertainty and the market mechanism", Quarterly Journal of Economics 84, pp. 488-500.

Guha, R. and M. Waldman, 1997. "Leasing solves the lemons problem", Johnson Graduate School of Management, Cornell University, mimeo.

Hendel, I. and A. Lizzeri, 1999a. "Interfering with Secondary Markets", Rand Journal of Economics, 30:1-21.

Hendel, I. and A. Lizzeri, 1999b. "Adverse Selection in Durable Goods Markets", American Economic Review 89(5): 1095-1115.

Janssen, M. and S. Roy, 1999a. "Trading a Durable Good in a Walrasian Market with Asymmetric Information", Tinbergen Institute Discussion Paper TI 98-015/1.

Janssen, M. and S. Roy, 1999b. "On the Nature of the lemons problem in Durable Goods Markets", Florida International University Working Paper 99-4.

Lizzeri, A., 1999. "Information Revelation and Certification Intermediaries", Rand Journal of Economics, forthcoming.

Sobel, J., 1991, "Durable goods monopoly with entry of new consumers", Econometrica 59(5), pp. 1455-85.

Taylor, C., 1999. "Time-on-the-market as a signal of quality", Review of Economic Studies 66, pp. 555-578. 
Vettas, N., 1997. "On the Informational Role of Quantities: Durable Goods and Consumers' word-of-mouth Communication", International Economic Review 38, pp. 915-944.

Vincent, D. R., 1990. "Dynamic auctions", Review of Economic Studies 57, pp. $49-61$.

Waldman, M., 1999. "Leasing, Lemons and Moral Hazard", Johnson Graduate School of Management, Cornell University, mimeo.

Wilson, C., 1979. "Equilibrium and adverse selection", American Economic Review 69, pp. 313-317.

Wilson, C., 1980. "The nature of equilibrium in markets with adverse selection", Bell Journal of Economics 11, pp. 108-130

\section{Appendix.}

From now on we will use the following notation.

a) $\quad K_{\tau}=\frac{\eta\left(\theta_{\tau-1}, \theta_{\tau}\right)-\eta\left(\theta_{\tau-1}, \theta_{\tau-1}\right)}{\left(\theta_{\tau}-\theta_{\tau-1}\right)}$

b) $F_{\tau}=\frac{\mu\left(\theta_{\tau-1}, \theta_{\tau}\right)}{\left(\theta_{\tau}-\theta_{\tau-1}\right)}$

c) $y_{\tau}=\theta_{\tau}-\theta_{\tau-1}$;

d) $z_{\tau}=y_{\tau}-y_{\tau-1}$;

e) $\gamma_{\tau}=\frac{1}{\delta} s_{\tau}$

f) $g(\theta)=\left\{\begin{array}{c}\frac{1}{\theta-\theta_{S}}(\theta-\nu \eta(\underline{\theta}, \theta)), \text { if } \theta \neq \theta_{S} \\ \lim _{\theta \rightarrow \theta_{S}} g(\theta)=1-a, \text { if } \theta=\theta_{S}\end{array}\right.$;

g) $\bar{g}(\theta)=\max _{\theta \in\left[\theta_{S}, \bar{\theta}\right]}|g(\theta)|$;

h) $\varphi_{t-1}=\theta_{t-1}(v-1)-\gamma_{t-1}$. 
Proof of Lemma 2.1 is on request.

Proof of Proposition 3.1. We prove all statements of the proposition sequentially.

a) Let us take any period $t$ of positive amount of trade $J_{t}$ so that $\mu\left(J_{t}\right)>0$ and take any $i \in J_{t}$. By the definition of dynamic equilibrium we can write:

$$
t \in \underset{\tau \geq t_{i}}{\arg \max }\left\{\left(p_{\tau}-\theta_{i}\right) \delta^{\tau-t_{i}} \mid\left(p_{\tau}-\theta_{i}\right) \geq 0\right\}
$$

This implies $\left(p_{t}-\theta_{i}\right) \delta^{t-t_{i}} \geq\left(p_{\tau}-\theta_{i}\right) \delta^{\tau-t_{i}}$ for all $\tau>t \geq t_{i}$. Now take any $\theta<\theta_{i}$ :

$$
\begin{aligned}
\left(p_{t}-\theta\right) \delta^{t-t_{i}}-\left(p_{\tau}-\theta\right) \delta^{\tau-t_{i}} & =\left(p_{t}-\theta_{i}\right) \delta^{t-t_{i}}-\left(p_{\tau}-\theta_{i}\right) \delta^{\tau-t_{i}}+\left(\theta_{i}-\theta\right)\left(\delta^{t}-\delta^{\tau}\right) \delta^{-t_{i}} \geq \\
& \geq\left(\theta_{i}-\theta\right)\left(1-\delta^{\tau-t}\right) \delta^{t-t_{i}}>0 .
\end{aligned}
$$

So, for all sellers with a good of quality less then $\theta_{i}$ who are still in the market in a certain period and have not yet traded it is optimal to trade in that period. Thus, we can define $\theta_{t}$ as $\theta_{t}=\sup \left\{\theta_{i} \mid i \in J_{t}\right\}$ and then it is easy to see that $J_{t}=\left\{i \theta_{i} \in\left[\underline{\theta}, \theta_{t}\right], t_{i} \leq t\right\}$. Finally, if $\mu\left(J_{t}\right)=0$ for some $t$, then we set $\theta_{t}=\underline{\theta}$.

b) By the equilibrium definition, for all $t \quad E_{t} \geq \underline{\theta}$ and $p_{t} \geq v E_{t}$ so that $p_{t} \geq v \underline{\theta}$. Thus, if $\mu\left(J_{t}\right)=0$, we have $p_{t} \geq \nu \underline{\theta}>\underline{\theta}=\theta_{t}$. If $\mu\left(J_{t}\right)>0$, it is optimal for the marginal seller $\theta_{t}$ to trade in period $t$ and a necessary condition is $p_{t}-\theta_{t} \geq 0$. So, $p_{t} \geq \theta_{t}$ for all $t$.

c) Suppose $p_{t}-\theta_{t}-\delta^{(\tilde{t}-t)}\left(p_{\tilde{t}}-\theta_{t}\right)=\sigma>0$. Then, we can find a seller $i$ of quality $\theta_{i}=\theta_{t}+\frac{1}{2} \sigma$ such that $\theta_{t}<\theta_{i}<\theta_{\tilde{t}}$ and $t_{i} \leq t$, i.e., he is in the market by period $t$. By definition of $\left\{\theta_{t}\right\}$ he will trade in period $\tilde{t}$. But it can be shown that this is not optimal:

$\delta^{\left(\tilde{t}-t_{i}\right)}\left(p_{\tilde{t}}-\left(\theta_{t}+\frac{1}{2} \sigma\right)\right)-\delta^{\left(t-t_{i}\right)}\left(p_{t}-\left(\theta_{t}+\frac{1}{2} \sigma\right)\right)=\delta^{t-t_{i}}\left[-\sigma+\frac{1}{2} \sigma\left(1-\delta^{(\tilde{t}-t)}\right)\right]<0$.

So, it is not possible that $p_{t}-\theta_{t}>\delta^{(\tilde{t}(t)-t)}\left(p_{\tilde{t}(t)}-\theta_{t}\right)$. A similar argument shows that it is impossible to have $p_{t}-\theta_{t}<\delta^{(\tilde{t}(t)-t)}\left(p_{\tilde{t}(t)}-\theta_{t}\right)$.

d) Suppose $\bar{\theta}-\theta_{\hat{t}}=\sigma>0$. We will show that in this case $p_{\hat{t}}-\theta_{\hat{t}}=0$. Suppose not. Then it must be $p_{\hat{t}}-\theta_{\hat{t}}=\varepsilon>0$. Let us take a seller $i$ of quality 
$\theta_{i}=\theta_{\hat{t}}+\frac{1}{2} \min \{\varepsilon, \sigma\}$ such that $\theta_{\hat{t}}<\theta_{i}<\bar{\theta}$ and $t_{i}=\hat{t}$. By definition of $\left\{\theta_{t}\right\}$ he will never trade because for all $t \geq t_{i} \quad \theta_{t} \leq \max _{t \geq \hat{t}}\left\{\theta_{t}\right\}=\theta_{\hat{t}}<\theta_{i}$. If he, however, traded in period $\hat{t}$ he would get

$p_{\hat{t}}-\theta_{i}=p_{\hat{t}}-\theta_{\hat{t}}-\frac{1}{2} \min \{\varepsilon, \sigma\}=\varepsilon-\frac{1}{2} \min \{\varepsilon, \sigma\} \geq \frac{1}{2} \varepsilon>0$, which is a contradiction.

So, it must be the case that $\left(p_{\hat{t}(t)}-\theta_{\hat{t}(t)}\right)\left(\bar{\theta}-\theta_{\hat{t}(t)}\right)=0$.

Proof of Proposition 4.1. Using the fact that $\theta_{\tau}\left(\theta_{1}\right)>\theta_{\tau-1}\left(\theta_{1}\right)$ we express the expected quality sold in period $\tau$ in terms of $\mu\left(\theta_{\tau-1}, \theta_{\tau}\right)$ and $\eta\left(\theta_{\tau-1}, \theta_{\tau}\right)$ :

$$
\eta_{\tau}\left(\theta_{\tau-1}, \theta_{\tau}\right)=\frac{\tau \eta\left(\theta_{\tau-1}, \theta_{\tau}\right) \mu\left(\theta_{\tau-1}, \theta_{\tau}\right)+\eta\left(\underline{\theta}, \theta_{\tau-1}\right) \mu\left(\underline{\theta}, \theta_{\tau-1}\right)}{\tau \mu\left(\theta_{\tau-1}, \theta_{\tau}\right)+\mu\left(\underline{\theta}, \theta_{\tau-1}\right)}, \tau \geq 1, \theta_{0} \equiv \underline{\theta}
$$

Now we consider the indifference condition (3) with $p_{\tau}=v \eta_{\tau}$. It can be written for $\tau \geq 2$ as $\eta_{\tau}\left(\theta_{\tau-1}, \theta_{\tau}\right)-\eta_{\tau-1}\left(\theta_{\tau-2}, \theta_{\tau-1}\right)=\frac{1-\delta}{v \delta} s_{\tau-1}$.

The main part of the proof is by induction. At first we will prove that if all conditions to be proved (except $\left.\theta_{t}\left(\theta_{1}\right)>\theta_{S}\right)$ are true for some $t>2$, then $\exists \theta_{t+1}\left(\theta_{1}\right)>\theta_{t}\left(\theta_{1}\right)$ such that those conditions are also true for $t+1$. Next, we will show that there exist $\theta_{1}$ and $\theta_{2}$ such that those conditions are satisfied. Finally, we will show that for some $T_{0}$ we get $\theta_{T_{0}}\left(\theta_{1}\right)>\theta_{S}$ and, therefore, $\theta_{t}\left(\theta_{1}\right)>\theta_{S}$ for all $t>T_{0}$ and all $\theta_{1} \in U_{t}^{0} \subset U_{T_{0}}^{0}$

Suppose that for some $t>2 \exists U_{t-1}^{0}=\left(\theta_{1}^{0}(t-1), \theta_{S}\right), \exists\left\{\theta_{\tau}\left(\theta_{1}\right)\right\}_{\tau=1}^{t-1}$, and $\exists\left\{p_{\tau}\left(\theta_{1}\right)\right\}_{\tau=1}^{t-1}$ such that for all $\theta_{1} \in U_{t-1}^{0}$ :
a) $\quad \theta_{\tau}\left(\theta_{1}\right)>\theta_{\tau-1}\left(\theta_{1}\right), s_{\tau}\left(\theta_{1}\right)>0 ; \theta_{\tau}\left(\theta_{S}\right)=\theta_{S}, s_{\tau}\left(\theta_{S}\right)=0$;
b) $\theta_{\tau}$ and $s_{\tau}$ are continuous functions differentiable at $\theta_{1}=\theta_{S}$ so that we can write:

$$
\theta_{\tau}=\theta_{S}+\frac{d \theta_{\tau}}{d \theta_{1}}\left(\theta_{S}\right)\left(\theta_{1}-\theta_{S}\right)+o\left(\theta_{1}-\theta_{S}\right), \text { and } s_{\tau}=\frac{d s_{\tau}}{d \theta_{1}}\left(\theta_{S}\right)\left(\theta_{1}-\theta_{S}\right)+o\left(\theta_{1}-\theta_{S}\right)
$$
c) $\frac{d s_{\tau}}{d \theta_{1}}\left(\theta_{S}\right)<0, \frac{d y_{\tau}}{d \theta_{1}}\left(\theta_{S}\right)<0, \frac{d z_{\tau}}{d \theta_{1}}\left(\theta_{S}\right)<0$; 
d) for all $\tau=1, \ldots, t-2 \quad p_{\tau}-\theta_{\tau}=\delta\left(p_{\tau+1}-\theta_{\tau}\right)$;

e) for all $\tau=1, \ldots, t-2 \frac{d\left(s_{\tau}-\beta s_{\tau-1}\right)}{d \theta_{1}}\left(\theta_{S}\right) \leq 0$, where $\beta=\frac{2 a-(1-\delta)}{2 a \delta}$.

We will prove that then $\exists U_{t}^{0}=\left(\theta_{1}^{0}(t), \theta_{S}\right) \subset U_{t-1}^{0}$ such that for all $\theta_{1} \in U_{t}^{0}$ equation (12) determines a unique value of $\theta_{t}$ as a function of $\theta_{1}$ and

a) $\theta_{t}\left(\theta_{1}\right)>\theta_{t-1}\left(\theta_{1}\right), s_{t}\left(\theta_{1}\right)>0 ; \theta_{t}\left(\theta_{S}\right)=\theta_{S}, s_{t}\left(\theta_{S}\right)=0$;

b) $\theta_{t}$ and $s_{t}$ are continuous functions differentiable at $\theta_{1}=\theta_{S}$ so that we can write:

$\theta_{t}=\theta_{S}+\frac{d \theta_{t}}{d \theta_{1}}\left(\theta_{S}\right)\left(\theta_{1}-\theta_{S}\right)+o\left(\theta_{1}-\theta_{S}\right)$, and $s_{t}=\frac{d s_{t}}{d \theta_{1}}\left(\theta_{S}\right)\left(\theta_{1}-\theta_{S}\right)+o\left(\theta_{1}-\theta_{S}\right) ;$

c) $\frac{d s_{t}}{d \theta_{1}}\left(\theta_{S}\right)<0, \frac{d y_{t}}{d \theta_{1}}\left(\theta_{S}\right)<0, \frac{d z_{t}}{d \theta_{1}}\left(\theta_{S}\right)<0$;

d) $\frac{d\left(s_{t}-\beta s_{t-1}\right)}{d \theta_{1}}\left(\theta_{S}\right)<0$, where $\beta=\frac{2 a-(1-\delta)}{2 a \delta}$, and $\theta_{t}\left(\theta_{1}\right)-\theta_{S}<s_{t}\left(\theta_{1}\right) \frac{2}{2 a-1}$.

Let us first consider the left-hand side of the equation (12) as a function $G_{t}\left(\theta_{t}, \theta_{t-1}, \theta_{t-2}\right) \equiv \eta_{t}\left(\theta_{t-1}, \theta_{t}\right)-\eta_{t-1}\left(\theta_{t-2}, \theta_{t-1}\right)$. It is easily seen that as $\theta_{t-1} \geq \theta_{t-2} \geq \underline{\theta}$ evaluating $G_{t}\left(\theta_{t}, \theta_{t-1}, \theta_{t-2}\right) \quad$ at $\quad \theta_{t}=\theta_{t-1} \quad$ yields $\quad G_{t}\left(\theta_{t-1}, \theta_{t-1}, \theta_{t-2}\right) \leq 0 . \quad$ So, $G_{t}\left(\theta_{t-1}\left(\theta_{1}\right), \theta_{t-1}\left(\theta_{1}\right), \theta_{t-2}\left(\theta_{1}\right)\right) \leq 0<\frac{1-\delta}{v \delta} s_{t-1}\left(\theta_{1}\right)$ for any $\theta_{1} \in U_{t-1}^{0}$. Also, for any small $\varepsilon>0 \quad$ such that $\theta_{S}+\varepsilon<\bar{\theta}, \quad G_{t}\left(\theta_{S}+\varepsilon, \theta_{t-1}\left(\theta_{S}\right), \theta_{t-2}\left(\theta_{S}\right)\right)>0$. As $G_{t}\left(\theta_{S}+\varepsilon, \theta_{t-1}\left(\theta_{S}\right), \theta_{t-2}\left(\theta_{S}\right)\right)>\frac{1-\delta}{v \delta} s_{t-1}\left(\theta_{S}\right)=0$ and as $G_{t}\left(\theta_{1}\right)$ and $s_{t-1}\left(\theta_{1}\right)$ are both continuous functions, there must exist a neighborhood $\overline{U_{t}^{0}}=\left(\overline{\theta_{1}^{0}(t)}, \theta_{S}\right) \subset U_{t-1}^{0}$ such that for any $\theta_{1} \in \overline{U_{t}^{0}} G_{t}\left(\theta_{S}+\varepsilon, \theta_{t-1}\left(\theta_{1}\right), \theta_{t-2}\left(\theta_{1}\right)\right)>\frac{1-\delta}{v \delta} s_{t-1}\left(\theta_{1}\right)$. Finally, $G_{t}\left(\theta_{t}, \theta_{t-1}, \theta_{t-2}\right)$ is a strictly increasing continuous function w.r.t. $\theta_{t}$.

Taking all these facts together and applying the intermediate point theorem we can draw the following conclusion. For all $\theta_{1} \in \overline{U_{t}^{0}}$ there exists a unique continuous function $\theta_{t}\left(\theta_{1}\right)$ such that $\theta_{t-1}\left(\theta_{1}\right)<\theta_{t}\left(\theta_{1}\right)<\theta_{S}+\varepsilon . \quad s_{t}\left(\theta_{1}\right)=v \eta_{t}\left(\theta_{t-1}, \theta_{t}\right)-\theta_{t}$ is also continuous function and $\theta_{t}\left(\theta_{S}\right)=\theta_{S}, s_{t}\left(\theta_{S}\right)=0$. 
To prove the rest of step (b) of our induction step, we will now show that $\theta_{t}\left(\theta_{1}\right)$ and $s_{t}\left(\theta_{1}\right)$ both are differentiable functions at $\theta_{1}=\theta_{S}$. For all $\tau=2, \ldots, t$ the indifference condition (3) can be written as $\eta_{\tau}\left(\theta_{\tau-1}, \theta_{\tau}\right)=\frac{1}{\delta} \eta_{\tau-1}\left(\theta_{\tau-2}, \theta_{\tau-1}\right)-\frac{1-\delta}{v \delta} \theta_{\tau-1}$. Taking the first differentials of this identity w.r.t. $\theta_{1}$ at $\theta_{1}=\theta_{S}$, and using (11), we get:

$$
\begin{aligned}
& d_{S}\left(\frac{\tau \eta\left(\theta_{\tau-1}, \theta_{\tau}\right) \mu\left(\theta_{\tau-1}, \theta_{\tau}\right)+\eta\left(\underline{\theta}, \theta_{\tau-1}\right) \mu\left(\underline{\theta}, \theta_{\tau-1}\right)}{\tau \mu\left(\theta_{\tau-1}, \theta_{\tau}\right)+\mu\left(\underline{\theta}, \theta_{\tau-1}\right)}\right)= \\
& \quad=\frac{1}{\delta} d_{S}\left(\frac{(\tau-1) \eta\left(\theta_{\tau-2}, \theta_{\tau-1}\right) \mu\left(\theta_{\tau-2}, \theta_{\tau-1}\right)+\eta\left(\underline{\theta}, \theta_{\tau-2}\right) \mu\left(\underline{\theta}, \theta_{\tau-2}\right)}{(\tau-1) \mu\left(\theta_{\tau-2}, \theta_{\tau-1}\right)+\mu\left(\underline{\theta}, \theta_{\tau-2}\right)}\right)-\frac{1-\delta}{v \delta} d_{S} \theta_{\tau-1},
\end{aligned}
$$

where $d_{S} \theta_{\tau}=\left.d \theta_{\tau}\right|_{\theta_{1}=\theta_{S}}$. Taking the derivatives explicitly into account yields

$$
\begin{aligned}
& \frac{\tau\left(f \theta_{S} d_{S} \theta_{\tau}-f \theta_{S} d_{S} \theta_{\tau-1}\right)+f \theta_{S} d_{S} \theta_{\tau-1}}{\tau \mu\left(\theta_{S}, \theta_{S}\right)+\mu\left(\underline{\theta}, \theta_{S}\right)}-\eta_{\tau}\left(\theta_{S}, \theta_{S}\right) \frac{\tau\left(f d_{S} \theta_{\tau}-f d_{S} \theta_{\tau-1}\right)+f d_{S} \theta_{\tau-1}}{\tau \mu\left(\theta_{S}, \theta_{S}\right)+\mu\left(\underline{\theta}, \theta_{S}\right)}= \\
& =\frac{1}{\delta}\left(f \theta_{S} \frac{(\tau-1) d_{S} \theta_{\tau-1}-(\tau-2) d_{S} \theta_{\tau-2}}{(\tau-1) \mu\left(\theta_{S}, \theta_{S}\right)+\mu\left(\underline{\theta}, \theta_{S}\right)}-f \eta_{\tau-1}\left(\theta_{S}, \theta_{S}\right) \frac{(\tau-1) d_{S} \theta_{\tau-1}-(\tau-2) d_{S} \theta_{\tau-2}}{(\tau-1) \mu\left(\theta_{S}, \theta_{S}\right)+\mu\left(\underline{\theta}, \theta_{S}\right)}\right)- \\
& \quad-\frac{1-\delta}{v \delta} d_{S} \theta_{\tau-1} .
\end{aligned}
$$

Rewriting gives

$$
\begin{aligned}
& \delta a\left(\tau d_{S} \theta_{\tau}-(\tau-1) d_{S} \theta_{\tau-1}\right)=a\left((\tau-1) d_{S} \theta_{\tau-1}-(\tau-2) d_{S} \theta_{\tau-2}\right)-(1-\delta) d_{S} \theta_{\tau-1}, \text { and } \\
& \tau \delta d_{S} \theta_{\tau}=d_{S} \theta_{\tau-1}\left((1+\delta)(\tau-1)-\frac{1-\delta}{a}\right)-(\tau-2) d_{S} \theta_{\tau-2} .
\end{aligned}
$$

So we can write $\frac{d \theta_{\tau}}{d \theta_{1}}\left(\theta_{S}\right)=\frac{(1+\delta)(\tau-1)-\frac{1-\delta}{a}}{\tau \delta} \frac{d \theta_{\tau-1}}{d \theta_{1}}\left(\theta_{S}\right)-\frac{\tau-2}{\tau \delta} \frac{d \theta_{\tau-2}}{d \theta_{1}}\left(\theta_{S}\right)$. As, by assumption, $\theta_{t-1}$ and $\theta_{t-2}$ both are differentiable at $\theta_{1}=\theta_{S}$ so is $\theta_{t}\left(\theta_{1}\right)$. Also, as surplus is defined by $s_{t}=p_{t}-\theta_{t}=v \eta_{t}\left(\theta_{t}, \theta_{t-1}\right)-\theta_{t}, s_{t}$ is also differentiable at $\theta_{1}=\theta_{S}$.

Next, we prove part (c) of the induction argument. To this end, we can rewrite (13), using (7) and (8) in the following way:

$$
\begin{aligned}
& \tau \delta d_{S} y_{\tau}=d_{S} y_{\tau-1}\left(\tau-1-\delta-\frac{1-\delta}{a}\right)-\frac{(1-\delta)(1-a)}{a} d_{S} \theta_{\tau-2}, \text { and } \\
& \tau \delta d_{S} z_{\tau}=d_{S} z_{\tau-1}\left(\tau-3+\frac{(1-\delta)(2 a-1)}{a}\right)+d_{S} y_{\tau-2} \frac{(1-\delta)(2 a-1)}{a} .
\end{aligned}
$$


Using the induction assumptions, we have $\frac{d y_{t-2}}{d \theta_{1}}\left(\theta_{S}\right)<0, \quad \frac{d y_{t-1}}{d \theta_{1}}\left(\theta_{S}\right)<0$ and $\frac{d z_{t-1}}{d \theta_{1}}\left(\theta_{S}\right)<0 \quad$ so $\quad$ it $\quad$ follows from $\quad(14)$ that $\quad \frac{d z_{t}}{d \theta_{1}}\left(\theta_{S}\right)<0, \quad$ and $\frac{d y_{t}}{d \theta_{1}}\left(\theta_{S}\right)=\frac{d y_{t-1}}{d \theta_{1}}\left(\theta_{S}\right)+\frac{d z_{t}}{d \theta_{1}}\left(\theta_{S}\right)<0$

Similarly, we can get the following expressions for the first differentials of the surpluses $d_{S} s_{t}$ :

$$
\begin{aligned}
& d_{S} s_{\tau}=a(\tau-1) d_{S} y_{\tau}-(1-a) d_{S} \theta_{\tau}, \text { and } \\
& d_{S}\left(s_{\tau}-s_{\tau-1}\right)=d_{S} z_{\tau}(a \tau-1)+d_{S} y_{\tau-1}(2 a-1) .
\end{aligned}
$$

Again, by our induction assumptions we have $\frac{d s_{t-1}}{d \theta_{1}}\left(\theta_{S}\right)<0$ and it follows that $\frac{d\left(s_{t}-s_{t-1}\right)}{d \theta_{1}}\left(\theta_{S}\right)<0$, and $\frac{d s_{t}}{d \theta_{1}}\left(\theta_{S}\right)=\frac{d s_{t-1}}{d \theta_{1}}\left(\theta_{S}\right)+\frac{d\left(s_{t}-s_{t-1}\right)}{d \theta_{1}}\left(\theta_{S}\right)<0$.

The only parts of the induction argument we still have to prove are $\frac{d\left(s_{t}-\beta s_{t-1}\right)}{d \theta_{1}}\left(\theta_{S}\right)<0$ and for all $t \exists U_{t}^{0}=\left(\theta_{1}^{0}(t), \theta_{S}\right) \in \overline{U_{t}^{0}}$ such that for all $\theta_{1} \in U_{t}^{0}$ $s_{t}\left(\theta_{1}\right)>\beta s_{t-1}\left(\theta_{1}\right)$, where $\beta=\frac{2 a-(1-\delta)}{2 a \delta}$.

Subtracting (15) for $\tau-1$ from (15) for $\tau$ we can write

$$
\begin{aligned}
& d_{S} s_{\tau}-d_{S} s_{\tau-1}=a(\tau-1) d_{S} y_{\tau}-a(\tau-2) d_{S} y_{\tau-1}-(1-a)\left(d_{S} \theta_{\tau}-d_{S} \theta_{\tau-1}\right), \text { or } \\
& d_{S} s_{\tau}-d_{S} s_{\tau-1}=(a \tau-1) d_{S} y_{\tau}-a(\tau-2) d_{S} y_{\tau-1} .
\end{aligned}
$$

We can write the indifference condition (3) as $s_{\tau-1}=\delta s_{\tau}+\delta\left(\theta_{\tau}-\theta_{\tau-1}\right)$, the first differentials of which w.r.t. $\theta_{1}$ at $\theta_{1}=\theta_{S}$ becomes $\delta d_{S} y_{\tau}=d_{S} s_{\tau-1}-\delta d_{S} s_{\tau}$. Substituting it into (17) yields $d_{S} s_{\tau}-d_{S} s_{\tau-1}=(a \tau-1)\left(d_{S} s_{\tau-1}-\delta d_{S} s_{\tau}\right)-a(\tau-2)\left(d_{S} s_{\tau-2}-\delta d_{S} s_{\tau-1}\right)$. Rewriting gives

$$
\tau \delta d_{S} s_{\tau}=\left((\tau-1)(1+\delta)+\frac{(1-\delta)(a-1)}{a}\right) d_{S} s_{\tau-1}-(\tau-2) d_{S} s_{\tau-2} .
$$

We can express the above equation in terms of $d_{S}\left(s_{\tau}-\beta s_{\tau-1}\right)$ instead of $d_{S} s_{\tau}$, where $\beta=\frac{2 a-(1-\delta)}{2 a \delta}$. It can be shown that 


$$
\tau \delta d_{S}\left(s_{\tau}-\beta s_{\tau-1}\right)=(\tau-2)\left[d_{S}\left(s_{\tau-1}-\beta s_{\tau-2}\right)\left(\delta+\frac{(1-\delta)}{2 a}\right)+\frac{(1-\delta)}{2 a} \frac{(2 a-1)(1-\delta)}{2 a \delta} d_{S} s_{\tau-2}\right] .
$$

By our induction assumptions we have $\frac{d\left(s_{t-1}-\beta s_{t-2}\right)}{d \theta_{1}}\left(\theta_{S}\right) \leq 0$, and $\frac{d s_{t-2}}{d \theta_{1}}\left(\theta_{S}\right)<0$ so it follows that $\frac{d\left(s_{t}-\beta s_{t-1}\right)}{d \theta_{1}}\left(\theta_{S}\right)<0$, and, therefore,

$$
\frac{d s_{t}}{d \theta_{1}}\left(\theta_{S}\right)<\beta \frac{d s_{t-1}}{d \theta_{1}}\left(\theta_{S}\right) \leq \cdots \leq \beta^{t-\tau} \frac{d s_{\tau}}{d \theta_{1}}\left(\theta_{S}\right) \leq \cdots \leq \beta^{t-1} \frac{d s_{1}}{d \theta_{1}}\left(\theta_{S}\right)<0 .
$$

Now again let us consider the indifference equation (3) as $\delta\left(\theta_{\tau}-\theta_{\tau-1}\right)=s_{\tau-1}-\delta s_{\tau}$. Summing it up from $\tau=2$ to $\tau=t$ and rewriting gives

$$
\theta_{t}=\theta_{1}+\frac{1}{\delta}\left(s_{1}-s_{t}\right)+\frac{1-\delta}{\delta} \sum_{2}^{t} s_{\tau}
$$

Taking the first differential w.r.t. $\theta_{1}$ at $\theta_{S}$ and using (19), we get:

$$
\begin{aligned}
\frac{d \theta_{t}}{d \theta_{1}}\left(\theta_{S}\right) & =1+\frac{1}{\delta}\left(\frac{d s_{1}}{d \theta_{1}}\left(\theta_{S}\right)-\frac{d s_{t}}{d \theta_{1}}\left(\theta_{S}\right)\right)+\frac{1-\delta}{\delta} \sum_{2}^{t} \frac{d s_{\tau}}{d \theta_{1}}\left(\theta_{S}\right)> \\
& >1+\frac{1}{\delta}\left(\beta^{-(t-1)} \frac{d s_{t}}{d \theta_{1}}\left(\theta_{S}\right)-\frac{d s_{t}}{d \theta_{1}}\left(\theta_{S}\right)\right)+\frac{1-\delta}{\delta} \sum_{2}^{t} \beta^{-(t-\tau)} \frac{d s_{t}}{d \theta_{1}}\left(\theta_{S}\right)= \\
& =1+\frac{1}{\delta}\left(\left(\beta^{-(t-1)}-1\right)+(1-\delta) \beta^{-t} \frac{\beta^{2}\left(\beta^{t-1}-1\right)}{\beta-1}\right) \frac{d s_{t}}{d \theta_{1}}\left(\theta_{S}\right)= \\
& =1+\frac{\left(1-\beta^{-(t-1)}\right)}{\delta}\left((1-\delta) \frac{\beta}{\beta-1}-1\right) \frac{d s_{t}}{d \theta_{1}}\left(\theta_{S}\right)>1+\frac{1}{\delta}\left((1-\delta) \frac{\beta}{\beta-1}-1\right) \frac{d s_{t}}{d \theta_{1}}\left(\theta_{S}\right)= \\
& =1+\frac{1}{\delta}\left(\frac{2 a-(1-\delta)-2 a+1}{2 a-1}\right) \frac{d s_{t}}{d \theta_{1}}\left(\theta_{S}\right)=1+\frac{1}{2 a-1} \frac{d s_{t}}{d \theta_{1}}\left(\theta_{S}\right)>\frac{1}{2 a-1} \frac{d s_{t}}{d \theta_{1}}\left(\theta_{S}\right) .
\end{aligned}
$$

As $\theta_{t}$ and $s_{t}$ are differentiable at $\theta_{1}=\theta_{S}$ they can be written as follows:

$$
\theta_{t}\left(\theta_{1}\right)-\theta_{S}-\frac{2}{2 a-1} s_{t}\left(\theta_{1}\right)=\left(\frac{d \theta_{t}}{d \theta_{1}}\left(\theta_{S}\right)-\frac{2}{2 a-1} \frac{d s_{t}}{d \theta_{1}}\left(\theta_{S}\right)\right)\left(\theta_{1}-\theta_{S}\right)+o\left(\theta_{1}-\theta_{S}\right) .
$$

The above inequality implies that $\exists U_{t}^{0}=\left(\theta_{1}^{0}(t), \theta_{S}\right) \in \overline{U_{t}^{0}}$ such that for all $\theta_{1} \in U_{t}^{0}$

$$
\begin{aligned}
& \theta_{t}\left(\theta_{1}\right)-\theta_{S}-\frac{2}{2 a-1} s_{t}\left(\theta_{1}\right)<-\frac{1}{2 a-1} \frac{d s_{t}}{d \theta_{1}}\left(\theta_{S}\right)\left(\theta_{1}-\theta_{S}\right)+o\left(\theta_{1}-\theta_{S}\right)<0, \text { or } \\
& \theta_{t}\left(\theta_{1}\right)-\theta_{S}<\varepsilon_{\gamma} s_{t}\left(\theta_{1}\right) \text {, where } \varepsilon_{\gamma}=\frac{1}{2 a-1} .
\end{aligned}
$$

Now we will show that all induction assumptions are valid for $t=1,2$. Let us first consider the function $s_{1}\left(\theta_{1}\right)=p_{1}-\theta_{1}=\nu \eta\left(\underline{\theta}, \theta_{1}\right)-\theta_{1}$. In terms of the function $\mu$ it can 
be written as $s_{1}\left(\theta_{1}\right)=\frac{v}{\mu\left(\underline{\theta}, \theta_{1}\right)} \int_{\underline{\theta}}^{\theta_{1}} \theta d \mu-\theta_{1} . \quad s_{1}\left(\theta_{1}\right)$ is a continuous function over $U_{0}^{0} \equiv\left(\theta_{S}-\varepsilon_{\mu}, \theta_{S}\right)$ and differentiable at $\theta_{1}=\theta_{S}$. From the definition of $a$ it follows that $\frac{d s_{1}}{d \theta_{1}}\left(\theta_{S}\right)=-(1-a)$ and $s_{1}\left(\theta_{1}\right)=-(1-a)\left(\theta_{1}-\theta_{S}\right)+o\left(\theta_{1}-\theta_{S}\right)$. Hence, there exists a neighborhood, namely $U_{1}^{0}=\left(\theta_{1}^{0}(1), \theta_{S}\right) \subset U_{0}^{0}$, such that for all $\theta_{1} \in U_{1}^{0} \quad s_{1}\left(\theta_{1}\right)>0$. Obviously, $\theta_{1}$ itself is continuous and differentiable at $\theta_{1}=\theta_{S}$. Then, using the definitions of $y_{\tau}$ and $z_{\tau}$, we get $\frac{d y_{2}}{d \theta_{1}}\left(\theta_{S}\right)=\frac{d \theta_{2}}{d \theta_{1}}\left(\theta_{S}\right)-1=-\frac{(1-\delta)(1-a)}{2 \delta a}<0, \quad$ and $\frac{d z_{2}}{d \theta_{1}}\left(\theta_{S}\right)=-\frac{(1-\delta)(1-a)}{2 \delta a}-1<0$

Finally, using (13), (15) and (16) yields $\frac{d\left(s_{2}-s_{1}\right)}{d \theta_{1}}\left(\theta_{S}\right)=-\frac{(1-\delta)(1-a)(2 a-1)}{2 \delta a}<0$, and, consequently, $\frac{d s_{2}}{d \theta_{1}}\left(\theta_{S}\right)<0$ and $\frac{d\left(s_{2}-\beta s_{1}\right)}{d \theta_{1}}\left(\theta_{S}\right)=0$. That ends the proof of the induction argument.

We finish the proof by showing that for some $T_{0}$ we must have $\theta_{T_{0}}\left(\theta_{1}\right)>\theta_{S}$, hence, for all $t>T_{0}$ and all $\theta_{1} \in U_{t}^{0} \subset U_{T_{0}}^{0}: \theta_{t}\left(\theta_{1}\right)>\theta_{S}$. To see this consider the sequence $\left\{\frac{d \theta_{\tau}}{d \theta_{1}}\left(\theta_{S}\right)\right\}_{\tau=1}^{\infty}$. The first term of this sequence equals 1. Moreover, the sequence is decreasing with strictly negative increment as

$$
\frac{d \theta_{\tau}}{d \theta_{1}}\left(\theta_{S}\right)-\frac{d \theta_{\tau-1}}{d \theta_{1}}\left(\theta_{S}\right)=\frac{d y_{\tau}}{d \theta_{1}}\left(\theta_{S}\right)<\frac{d y_{2}}{d \theta_{1}}\left(\theta_{S}\right)=-\frac{(1-\delta)(1-a)}{2 \delta a}<0 .
$$

Thus, there exists a first negative term of $\left\{\frac{d \theta_{\tau}}{d \theta_{1}}\left(\theta_{S}\right)\right\}_{\tau=1}^{\infty}$, which can be denoted by $\frac{d \theta_{T_{0}}}{d \theta_{1}}\left(\theta_{S}\right)<0$, where $T_{0} \leq\left[\frac{2 \delta a}{(1-\delta)(1-a)}+1\right]$. It implies that for all $\theta_{1} \in U_{T_{0}}^{0} \quad \theta_{S}<\theta_{T_{0}}\left(\theta_{1}\right)$ 


\section{Proof of Proposition 4.2.}

In case when $\theta_{t}>\theta_{t-1}$ we have $\tilde{t}(\tau-1)=\tau$ and our indifference equation (2) can be written as (3), or $\quad p_{\tau}=\theta_{\tau-1}+\frac{1}{\delta}\left(p_{\tau-1}-\theta_{\tau-1}\right)=\frac{1}{\delta} s_{\tau-1}+\theta_{\tau-1} . \quad$ Rewriting yields $\nu \eta_{t}\left(\theta_{t-1}, \theta_{t}\right)=\frac{1}{\delta} s_{t-1}+\theta_{t-1}$.

Function $\eta_{t}\left(\theta_{t-1}, \theta_{t}\right)$ strictly increases w.r.t. $\theta_{t}$ for all $\theta_{t-1}$, so there exists an inverse function which determines $\theta_{t}$ as a function of $\theta_{t-1}\left(\theta_{1}\right)$ and $s_{t-1}\left(\theta_{1}\right)$, $\theta_{t}=\theta_{t}\left(\theta_{t-1}, \frac{1}{\delta} s_{t-1}\right)$. This function is defined for all $\theta_{1}$ as long as $v \eta_{t}\left(\theta_{t-1}\left(\theta_{1}\right), \bar{\theta}\right) \geq \frac{1}{\delta} s_{t-1}\left(\theta_{1}\right)+\theta_{t-1}\left(\theta_{1}\right)$. Using (11) we can write:

$$
v \frac{t \eta\left(\theta_{t-1}, \theta_{t}\right) \mu\left(\theta_{t-1}, \theta_{t}\right)+\eta\left(\underline{\theta}, \theta_{t-1}\right) \mu\left(\underline{\theta}, \theta_{t-1}\right)}{t \mu\left(\theta_{t-1}, \theta_{t}\right)+\mu\left(\underline{\theta}, \theta_{t-1}\right)}=\gamma_{t-1}+\theta_{t-1}, \text { where } \gamma_{t}=\frac{1}{\delta} s_{t} .
$$

Then

$$
\begin{aligned}
& y_{t}\left(\nu \eta\left(\theta_{t-1}, \theta_{t}\right)-\left(\gamma_{t-1}+\theta_{t-1}\right)\right)=\frac{\mu\left(\underline{\theta}, \theta_{t-1}\right)}{t \frac{\mu\left(\theta_{t-1}, \theta_{t}\right)}{y_{t}}}\left(\gamma_{t-1}+\frac{\theta_{t-1}-\nu \eta\left(\underline{\theta}, \theta_{t-1}\right)}{\theta_{t-1}-\theta_{S}}\left(\theta_{t-1}-\theta_{S}\right)\right), \text { and } \\
& y_{t}\left(\nu \eta\left(\theta_{t-1}, \theta_{t}\right)-\theta_{t-1}-\gamma_{t-1}\right)=\frac{\mu\left(\underline{\theta}, \theta_{t-1}\right)}{t F_{t}}\left(\gamma_{t-1}+g\left(\theta_{t-1}\right)\left(\theta_{t-1}-\theta_{S}\right)\right) .
\end{aligned}
$$

where $g$ and $F_{t}$ were defined in (9) and (6) correspondingly. Then we get:

$$
\begin{aligned}
& v y_{t}{ }^{2} \frac{\eta\left(\theta_{t-1}, \theta_{t}\right)-\theta_{t-1}}{y_{t}}+y_{t}\left((v-1) \theta_{t-1}-\gamma_{t-1}\right)=\frac{\mu\left(\underline{\theta}, \theta_{t-1}\right)}{t F_{t}}\left(\gamma_{t-1}+g\left(\theta_{t-1}\right)\left(\theta_{t-1}-\theta_{S}\right)\right) \text {, and } \\
& v y_{t}{ }^{2} K_{t}+y_{t} \varphi_{t-1}-\frac{\mu\left(\underline{\theta}, \theta_{t-1}\right)}{t F_{t}}\left(\gamma_{t-1}+g\left(\theta_{t-1}\right)\left(\theta_{t-1}-\theta_{S}\right)\right)=0,
\end{aligned}
$$

where $K_{t}$ and $\varphi_{t-1}$ were defined in (5) and (10) correspondingly.

Now let us take any $\varepsilon_{S}>0$ and any $\varepsilon_{\theta} \in\left(0, \min \left\{\varepsilon_{\gamma}, \frac{1}{2}\left(\bar{\theta}-\theta_{S}\right)\right\}\right)$. Then we take a small $\varepsilon>0$ such that $\varepsilon<\frac{1-\delta}{v \delta} \min \left\{1, \frac{\varepsilon_{S}}{2(v-1) \bar{\theta}}, \frac{1}{3 \bar{\theta}} \varepsilon_{\theta}\right\}$, a small $\varepsilon_{1}>0$ such that $\varepsilon_{1}<\frac{1}{2} \min \left\{\varepsilon_{S},(v-1) \theta_{S}\right\}, \quad$ and $\quad$ a $\quad$ large $T \quad T$ such that 
$T>\frac{\left(1+\bar{g} \varepsilon_{\gamma}\right) \mu(\underline{\theta}, \bar{\theta})}{m_{\mu}} \max \left\{\frac{1}{\delta \varepsilon \varepsilon_{1}}, \frac{4 v M_{\eta}}{\varepsilon_{1}^{2}}(v-1) \bar{\theta}, \frac{4(\bar{\theta}-\underline{\theta})}{m_{\eta}\left(\bar{\theta}-\theta_{S}\right)^{2}}\right\}$. By the assumption of the

Proposition 4.2 for that $T$ there exist corresponding $U_{T}^{0}=\left(\theta_{1}^{0}(T), \theta_{S}\right)$ and $\Theta_{T}\left(U_{T}^{0}\right)$.

Now let us take the subset $\hat{U}_{T}^{0}=\left(\hat{\theta_{1}^{0}}(T), \theta_{S}\right) \subset U_{T}^{0}$ such that for all $\theta_{1} \in \hat{U}_{T}^{0}$ :

a) $\theta_{1}-\theta_{S}+\frac{1}{\delta} s_{1}\left(\theta_{1}\right)<\frac{1}{3} \varepsilon_{\theta}$

b) $\theta_{T}-\theta_{S}<\varepsilon_{\theta}$

c) $\max _{\tau=1, \ldots, T}\left\{\sup _{\theta_{1} \in \hat{U}_{T}^{0}} s_{\tau}\left(\theta_{1}\right)\right\}<\frac{\varepsilon_{\theta}}{3} \frac{\delta}{(1-\delta)(T-1)}$;

d) $\varphi_{T}\left(\theta_{1}\right)>\varepsilon_{1}$ (it is always possible as $\varphi_{T}\left(\theta_{S}\right)=(v-1) \theta_{S}>\varepsilon_{1}$ ).

Now we will prove that if for all $\theta_{1} \in \hat{U}_{T}^{0}$ and some $t \geq T+1 \varphi_{t-1}\left(\theta_{1}\right)>\varepsilon_{1}$, then there exist well-defined functions $\theta_{t}\left(\theta_{1}\right)$ and $s_{t}\left(\theta_{1}\right)$ such that $y_{t}=\theta_{t}-\theta_{t-1}$ is determined by (20) and $s_{t}\left(\theta_{1}\right)>s_{t-1}\left(\frac{1}{\delta}-\varepsilon\right)>0$.

At first we prove the existence of $\theta_{t}\left(\theta_{1}\right)$ showing that $v \eta_{t}\left(\theta_{t-1}\left(\theta_{1}\right), \bar{\theta}\right) \geq \frac{1}{\delta} s_{t-1}\left(\theta_{1}\right)+\theta_{t-1}\left(\theta_{1}\right)$ if $\varphi_{t-1}\left(\theta_{1}\right) \geq 0$ and $\theta_{t-1}<\theta_{S}+\varepsilon_{\theta}$ :

$$
\begin{aligned}
& \nu \eta_{t}\left(\theta_{t-1}, \bar{\theta}\right)-\frac{1}{\delta} s_{t-1}+\theta_{t-1}=v \eta_{t}\left(\theta_{t-1}, \bar{\theta}\right)+\varphi_{t-1}-v \theta_{t-1} \geq v\left(\eta_{t}\left(\theta_{t-1}, \bar{\theta}\right)-\theta_{t-1}\right)= \\
& =v\left(\frac{m\left(\theta_{t-1}, \bar{\theta}\right) \mu\left(\theta_{t-1}, \bar{\theta}\right)+\eta\left(\underline{\theta}, \theta_{t-1}\right) \mu\left(\underline{\theta}, \theta_{t-1}\right)}{t \mu\left(\theta_{t-1}, \bar{\theta}\right)+\mu\left(\underline{\theta}, \theta_{t-1}\right)}-\theta_{t-1}\right)> \\
& >v \frac{t m_{\eta} m_{\mu}\left(\bar{\theta}-\theta_{t-1}\right)^{2}-(\bar{\theta}-\underline{\theta}) \mu(\underline{\theta}, \bar{\theta})}{t \mu\left(\theta_{t-1}, \bar{\theta}\right)+\mu\left(\underline{\theta}, \theta_{t-1}\right)}>v \frac{\frac{1}{4} T m_{\eta} m_{\mu}\left(\bar{\theta}-\theta_{s}\right)^{2}-(\bar{\theta}-\underline{\theta}) \mu(\underline{\theta}, \bar{\theta})}{t \mu\left(\theta_{t-1}, \bar{\theta}\right)+\mu\left(\underline{\theta}, \theta_{t-1}\right)}>0,
\end{aligned}
$$

thus there exist $\theta_{t}\left(\theta_{1}\right)$ and $s_{t}\left(\theta_{1}\right)$ such that $y_{t}=\theta_{t}-\theta_{t-1}$ is determined by (20).

Using the fact that for $t=T+1 \varphi_{t-1}\left(\theta_{1}\right)>\varepsilon_{1}$ we can solve (20) w.r.t. $y_{t}{ }^{9}$ :

$$
y_{t}=\frac{\varphi_{t-1}}{2 v K_{t}}\left[-1+\sqrt{1+\frac{4 v K_{t} \mu\left(\underline{\theta}, \theta_{t-1}\right)}{t F_{t} \varphi_{t-1}^{2}}\left(\gamma_{t-1}+g\left(\theta_{t-1}\right)\left(\theta_{t-1}-\theta_{S}\right)\right)}\right] \text {. }
$$

\footnotetext{
${ }^{9}$ Another solution is always negative and doesn't satisfy $\theta_{t}>\theta_{t-1}$.
} 
It can be shown that the expression under the square root above is positive and, therefore, $y_{t}$ is uniquely defined by (21).

Now we will show that $s_{t}>s_{t-1}\left(\frac{1}{\delta}-\varepsilon\right)$. Using the well-known inequality that for all $x>0 \sqrt{1+x}<1+\frac{1}{2} x$ we get:

$$
\begin{aligned}
y_{t} & =\frac{\varphi_{t-1}}{2 v K_{t}}\left[-1+\sqrt{1+\frac{4 v K_{t} \mu\left(\underline{\theta}^{\prime}, \theta_{t-1}\right)}{t F_{t} \varphi_{t-1}^{2}}\left(\gamma_{t-1}+g\left(\theta_{t-1}\right)\left(\theta_{t-1}-\theta_{S}\right)\right)}\right]< \\
& <\frac{\mu\left(\underline{\theta}, \theta_{t-1}\right)}{t F_{t} \varphi_{t-1}}\left(\gamma_{t-1}+g\left(\theta_{t-1}\right)\left(\theta_{t-1}-\theta_{S}\right)\right)<\frac{\mu(\underline{\theta}, \bar{\theta})}{T m_{\mu} \varepsilon_{1}}\left(1+\bar{g} \varepsilon_{\gamma}\right) \gamma_{t-1}<\delta \varepsilon \gamma_{t-1},
\end{aligned}
$$

and, therefore, $s_{t}=\frac{1}{\delta} s_{t-1}-y_{t}>\frac{1}{\delta} s_{t-1}-\varepsilon s_{t-1}=s_{t-1}\left(\frac{1}{\delta}-\varepsilon\right)$.

Now we will prove that $\exists T_{S}>T, \exists U_{T_{S}}^{S}=\left(\theta_{1}^{S}\left(T_{S}\right), \theta_{S}\right) \subset \hat{U}_{T}^{0}$ and $\exists \Theta_{T_{S}}$ such that for all $t=T+1, \ldots, T_{S}-1$ and for all $\theta_{1} \in U_{T_{S}}^{S} s_{t}>\left(\frac{1}{\delta}-\varepsilon\right) s_{t-1}, \varphi_{t-1}>\varepsilon_{1}$ and $\varphi_{T_{S}-1}\left(\theta_{1}^{S}\right)=\varepsilon_{1}$.

Suppose not, then for all $t \geq T+1$ and for all $\theta_{1} \in \hat{U}_{T}^{0} \varphi_{t-1}\left(\theta_{1}\right)>\varepsilon_{1}$. But in this case we have an induction: for all $t \geq T+1 \quad \exists \theta_{t}\left(\theta_{1}\right) \in\left(\theta_{t-1}\left(\theta_{1}\right), \bar{\theta}\right)$ and $\exists s_{t}\left(\theta_{1}\right)>\left(\frac{1}{\delta}-\varepsilon\right) s_{t-1}\left(\theta_{1}\right)>0$. Let fix any $\theta_{1} \in \hat{U}_{T}^{0}$ and consider the following sequences of numbers: $\left\{\theta_{t}\right\}_{t=T+1}^{\infty}$ and $\left\{s_{t}\right\}_{t=T+1}^{\infty}$. The former increases and is bounded, so $\exists \lim _{t \rightarrow \infty} \theta_{t}=\theta_{\infty} \leq \bar{\theta}$. The later also increases and $\exists \lim _{t \rightarrow \infty} s_{t}=+\infty$ as

$$
S_{t}>\left(\frac{1}{\delta}-\varepsilon\right) S_{t-1}>\left(\frac{1}{\delta}-\frac{1-\delta}{v \delta}\right) s_{t-1}=\left(1+\frac{(v-1)(1-\delta)}{\delta v}\right) s_{t-1} .
$$

But if we take a limit of $\varphi_{t-1}$ we get a contradiction:

$$
\lim _{t \rightarrow \infty} \varphi_{t}=\lim _{t \rightarrow \infty}\left((v-1) \theta_{t-1}-\frac{1}{\delta} S_{t-1}\right)=(v-1) \theta_{\infty}-\frac{1}{\delta} \lim _{t \rightarrow \infty} s_{t-1}=-\infty,
$$

as $\varphi_{t-1}>\varepsilon_{1}$ for all $t \geq T+1$.

So, it must be the case that $\exists T_{S}>T, \exists U_{T_{S}}^{S}=\left(\theta_{1}^{S}, \theta_{S}\right)$ and $\exists \Theta_{T_{S}}$ such that for all $t=T+1, \ldots, T_{S}-1$ and for all $\theta_{1} \in U_{T_{S}}^{S} s_{t}>\left(\frac{1}{\delta}-\varepsilon\right) s_{t-1}>0, \varphi_{t-1}>\varepsilon_{1}$ and $\varphi_{T_{S}-1}\left(\theta_{1}^{S}\right)=\varepsilon_{1}$.

Now we will prove by induction that for all $t \geq T_{S} \quad \exists U_{t}^{S}=\left(\theta_{1}^{S}(t), \theta_{S}\right) \subset U_{t-1}^{S}$ and $\exists \Theta_{t} \supset \Theta_{t-1}$ such that $\varphi_{t-1}\left(\theta_{1}^{S}(t)\right)=\varepsilon_{1}$ and $s_{t}\left(\theta_{1}\right)>\left(\frac{1}{\delta}-\varepsilon\right) s_{t-1}\left(\theta_{1}\right)$ for all $\theta_{1} \in U_{t}^{S}$. Suppose that for some $t \geq T_{S} \quad \exists U_{t}^{S}=\left(\theta_{1}^{S}(t), \theta_{S}\right) \subset U_{T_{S}}^{S}$ and $\exists \Theta_{t}$ such that for all 
$\tau=T+1, \ldots, t-1$ and for all $\theta_{1} \in U_{t}^{S} \quad \varphi_{\tau-1}\left(\theta_{1}\right)>\varepsilon_{1}, \quad s_{\tau}\left(\theta_{1}\right)>\left(\frac{1}{\delta}-\varepsilon\right) s_{\tau-1}\left(\theta_{1}\right)>0$ and $\varphi_{t-1}\left(\theta_{1}^{S}(t)\right)=\varepsilon_{1}$. It implies that

$$
\begin{aligned}
(v- & 1) \theta_{t}\left(\theta_{1}^{S}(t)\right)-s_{t}\left(\theta_{1}^{S}(t)\right)=\left(\theta_{t-1}+y_{t}\right)(v-1)-\left(\gamma_{t-1}-y_{t}\right)=(v-1) \theta_{t-1}-\gamma_{t-1}+v y_{t}= \\
& =\varepsilon_{1}+v y_{t}<\frac{1}{2} \varepsilon_{S}+v \varepsilon \delta \gamma_{t-1}=\frac{1}{2} \varepsilon_{S}+v \varepsilon \delta\left((v-1) \theta_{t-1}-\varphi_{t-1}\right)=\frac{1}{2} \varepsilon_{S}+v \varepsilon \delta(v-1) \theta_{t-1}< \\
& <\frac{1}{2} \varepsilon_{S}+v \varepsilon \delta(v-1) \bar{\theta}<\frac{1}{2} \varepsilon_{S}+\frac{1}{2} \varepsilon_{S}=\varepsilon_{S} .
\end{aligned}
$$

So, $s_{t}\left(\theta_{1}^{S}\right)>(v-1) \theta_{t}\left(\theta_{1}^{S}\right)-\varepsilon_{S}$.

Summing up the indifference equation (3) in a form $\delta\left(\theta_{\tau}-\theta_{\tau-1}\right)=s_{\tau-1}-\delta s_{\tau}$ from $\tau=2$ to $\tau=t$ we get $\delta\left(\theta_{t}-\theta_{1}\right)=\sum_{\tau=2}^{t} s_{\tau-1}-\delta \sum_{\tau=2}^{t} s_{\tau}=\left(s_{1}-s_{t}\right)+(1-\delta) \sum_{\tau=2}^{t} s_{\tau}$, or

$$
\begin{aligned}
\theta_{t}-\theta_{S} & =\left(\theta_{1}-\theta_{S}+\frac{1}{\delta} s_{1}\right)+\frac{1-\delta}{\delta} \sum_{\tau=2}^{t} s_{\tau}-\frac{1}{\delta} s_{t}=\frac{1}{3} \varepsilon_{\theta}+\frac{1}{\delta}\left((1-\delta) \sum_{\tau=2}^{t} s_{\tau}-s_{t}\right)= \\
& =\frac{1}{3} \varepsilon_{\theta}+\frac{1}{\delta}\left((1-\delta) \sum_{\tau=2}^{T} s_{\tau}+(1-\delta) \sum_{\tau=T+1}^{t} s_{\tau}-s_{t}\right)< \\
& <\frac{1}{3} \varepsilon_{\theta}+\frac{1}{\delta}\left((1-\delta)(T-1) \max _{\tau=1, \ldots, t}\left\{\sup s_{\tau}\right\}+(1-\delta) \sum_{\tau=T+1}^{t}\left(s_{t}\left(\frac{1}{\delta}-\varepsilon\right)^{\tau-t}\right)-s_{t}\right)< \\
& <\frac{1}{3} \varepsilon_{\theta}+\frac{1}{\delta}\left(\frac{\delta}{3} \varepsilon_{\theta}+(1-\delta) s_{t} \sum_{\tau=-\infty}^{t}\left(\frac{1}{\delta}-\varepsilon\right)^{\tau-t}-s_{t}\right)=\frac{2}{3} \varepsilon_{\theta}+\frac{1}{\delta}\left(\frac{(1-\delta)}{1-\left(\frac{1}{\delta}-\varepsilon\right)^{-1}}-1\right) s_{t}< \\
& <\frac{2}{3} \varepsilon_{\theta}+\frac{\delta \varepsilon}{1-\delta-\delta \varepsilon} \gamma_{t-1}=\frac{2}{3} \varepsilon_{\theta}+\frac{\delta \varepsilon}{1-\delta-\delta \varepsilon}\left((v-1) \theta_{t-1}-\varphi_{t-1}\right)<\frac{2}{3} \varepsilon_{\theta}+\frac{\varepsilon(v-1) \theta_{t-1}}{\frac{1-\delta}{\delta}-\varepsilon}< \\
& <\frac{2}{3} \varepsilon_{\theta}+\frac{\varepsilon(v-1) \bar{\theta}}{\frac{1-\delta}{\delta}-\frac{1-\delta}{v \delta}}=\frac{2}{3} \varepsilon_{\theta}+\frac{\varepsilon(v-1) \bar{\theta}}{\frac{1-\delta}{v \delta}(v-1)}=\frac{2}{3} \varepsilon_{\theta}+\frac{v \delta \varepsilon \bar{\theta}}{1-\delta}<\frac{2}{3} \varepsilon_{\theta}+\frac{1}{3} \varepsilon_{\theta}=\varepsilon_{\theta} .
\end{aligned}
$$

So, $\theta_{t}-\theta_{S}<\varepsilon_{\theta}$ for all $\theta_{1} \in U_{t}^{S}$.

Finally, let us consider $\varphi_{t}\left(\theta_{1}^{S}(t)\right)$ :

$$
\begin{aligned}
& \varphi_{t}\left(\theta_{1}^{S}(t)\right)=(v-1) \theta_{t}\left(\theta_{1}^{S}(t)\right)-\frac{1}{\delta} s_{t}\left(\theta_{1}^{S}(t)\right)=\left(\theta_{t-1}+y_{t}\right)(v-1)-\frac{1}{\delta}\left(\gamma_{t-1}-y_{t}\right)= \\
& =\varphi_{t-1}+y_{t}\left(v-1+\frac{1}{\delta}\right)-\frac{1-\delta}{\delta} \gamma_{t-1}<\varepsilon_{1}+\delta \varepsilon \gamma_{t-1}\left(v-1+\frac{1}{\delta}\right)-\frac{1}{\delta} \gamma_{t-1}(1-\delta)< \\
& <\varepsilon_{1}+(\delta v \varepsilon-(1-\delta)) \frac{1}{\delta} \gamma_{t-1}<\varepsilon_{1}+\left(\delta v \frac{1-\delta}{v \delta}-(1-\delta)\right) \frac{1}{\delta} \gamma_{t-1}=\varepsilon_{1},
\end{aligned}
$$

so $\varphi_{t}\left(\theta_{1}^{S}(t)\right)<\varepsilon_{1}$. On the other hand $\varphi_{t}\left(\theta_{S}\right)=(v-1) \theta_{S}>\varepsilon_{1}$ and $\varphi_{t}\left(\theta_{1}\right)$ is continuous, so $\exists \theta_{1}^{S}(t+1) \in U_{t}^{S}=\left(\theta_{1}^{S}(t), \theta_{S}\right)$ such that $\varphi_{t}\left(\theta_{1}^{S}(t+1)\right)=\varepsilon_{1}$, that ends the induction.

But if $\varphi_{t}\left(\theta_{1}^{S}(t+1)\right)=\varepsilon_{1}$ then we have $s_{t+1}\left(\theta_{1}^{S}(t+1)\right)>(v-1) \theta_{t+1}\left(\theta_{1}^{S}(t+1)\right)-\varepsilon_{S}$ as was shown above, and let us take $U_{t+1}^{S}=\left(\theta_{1}^{S}(t+1), \theta_{S}\right) \subset U_{t}^{S}$ so that $\theta_{t+1}-\theta_{S}<\varepsilon_{\theta}$ for all $\theta_{1} \in U_{t+1}^{S}$, that ends the proof. 


\section{Proof of Proposition 4.3.}

In all previous analysis we were considering $\theta_{t}$ as a function of $\theta_{1}, \theta_{t}=\theta_{t}\left(\theta_{1}\right)$. Now we will consider $\theta_{t}$ as a function of $\theta_{t-1}$ and $\gamma_{t-1}, \theta_{t}=\theta_{t}\left(\theta_{t-1}, \gamma_{t-1}\right)$, where $\gamma_{t-1}=\gamma_{t-1}\left(\theta_{1}\right)$ and $\theta_{t-1}=\theta_{t-1}\left(\theta_{1}\right)$. We define the following limit function:

$$
\hat{\theta_{1}}\left(\hat{\theta_{0}}, \hat{\gamma_{0}}\right)=\lim _{\tau \rightarrow \infty} \theta_{\tau}\left(\hat{\theta_{0}}, \hat{\gamma}_{0}\right) \text {. }
$$

In the same spirit as before we introduce functions $\hat{y}_{1}\left(\hat{\theta_{0}}, \hat{\gamma_{0}}\right)=\hat{\theta_{1}}\left(\hat{\theta_{0}}, \hat{\gamma_{0}}\right)-\hat{\theta_{0}}$, $\hat{s}_{1}\left(\hat{\theta_{0}}, \hat{\gamma_{0}}\right)=\hat{\gamma}_{0}-\hat{y}_{1}\left(\hat{\theta_{0}}, \hat{\gamma_{0}}\right), \hat{\gamma_{1}}\left(\hat{\theta_{0}}, \hat{\gamma_{0}}\right)=\frac{1}{\delta} \hat{s_{1}}\left(\hat{\theta_{0}}, \hat{\gamma_{0}}\right), \hat{\varphi_{0}}\left(\hat{\theta_{0}}, \hat{\gamma_{0}}\right)=(v-1) \hat{\theta_{0}}-\hat{\gamma_{0}}$.

Taking the limit (22) explicitly $^{10}$ yields that the limit actually exists for all $\hat{\theta_{0}} \in\left[\theta_{s}, \bar{\theta}-\varepsilon\right)$ and $\hat{\gamma_{0}} \in\left(\varepsilon, \nu \eta\left(\hat{\theta_{0}}, \bar{\theta}\right)-\hat{\theta_{0}}-\varepsilon\right)$ where $\varepsilon>0$ is an arbitrarily small number. Convergence is uniform, hence $\hat{\theta_{1}}\left(\hat{\theta_{0}}, \hat{\gamma}_{0}\right)$ is continuous and it follows that

$$
\hat{\theta_{1}}\left(\hat{\theta_{0}}, \hat{\gamma_{0}}\right)=\left\{\begin{array}{c}
\hat{\theta_{0}}, \text { if } \varepsilon<\hat{\gamma_{0}} \leq(v-1) \hat{\theta_{0}} \\
\hat{\theta_{1}}\left(\hat{\theta_{0}}, \hat{\gamma_{0}}\right): v \eta\left(\hat{\theta_{0}}, \hat{\theta_{1}}\right)=\hat{\theta_{0}}+\hat{\gamma_{0}}, \hat{\text { if }}(v-1) \hat{\theta_{0}} \leq \hat{\gamma_{0}}<v \eta \hat{\theta_{0}}, \hat{\theta}-\varepsilon .
\end{array}\right.
$$

Then we define $\hat{\theta_{1}}\left(\hat{\theta_{0}}, \hat{\gamma_{0}}\right)$ on a boundary where $\hat{\gamma}_{0}=0, \hat{\gamma_{0}}=\nu \eta\left(\hat{\theta_{0}}, \bar{\theta}\right)-\hat{\theta_{0}}$ or $\hat{\theta_{0}}=\bar{\theta}$ by taking corresponding limits of the function $\hat{\theta_{1}}\left(\hat{\theta_{0}}, \hat{\gamma_{0}}\right)$ when $\varepsilon \rightarrow+0$, that yields $\hat{\theta_{1}}\left(\hat{\theta_{0}}, 0\right)=\hat{\theta_{0}}, \hat{\theta_{1}}\left(\hat{\theta_{0}}, \nu \eta\left(\hat{\theta_{0}}, \bar{\theta}\right)-\hat{\theta_{0}}\right)=\bar{\theta}$ and $\hat{\theta_{1}}\left(\bar{\theta}, \hat{\gamma_{0}}\right)=\bar{\theta}{ }^{11}$.

Finally we define $\hat{\theta_{t+1}}\left(\hat{\theta_{0}}, \hat{\gamma_{0}}\right)$ for all $t>1$ as follows. If for some $\hat{\theta_{0}} \in\left[\theta_{s}, \bar{\theta}\right]$, $\hat{\gamma_{0}} \in\left[0, \frac{v-1}{\delta} \hat{\theta_{0}}\right]$ and for all $\tau=0, \ldots, t$ there exist functions $\hat{\theta_{\tau}}\left(\hat{\theta_{0}}, \hat{\gamma_{0}}\right)$ and $\hat{\gamma_{\tau}}\left(\hat{\theta_{0}}, \hat{\gamma_{0}}\right)$ such that $0 \leq \hat{\gamma}_{\tau} \leq v \eta\left(\hat{\theta_{\tau}}, \bar{\theta}\right)-\hat{\theta_{\tau}}$ and $\hat{\theta_{\tau}}\left(\hat{\theta_{0}}, \hat{\gamma_{0}}\right) \leq \bar{\theta}$ then we take $\hat{\theta_{t+1}}\left(\hat{\theta_{0}}, \hat{\gamma_{0}}\right)=\hat{\theta_{1}}\left(\hat{\theta_{t}}, \hat{\gamma_{t}}\right)$.

It can be easily seen that if $0<\hat{\gamma_{\tau}}<\nu \eta\left(\hat{\theta_{\tau}}, \bar{\theta}\right)-\hat{\theta_{\tau}}$ and $\hat{\theta_{\tau}}\left(\hat{\theta_{0}}, \hat{\gamma_{0}}\right)<\bar{\theta}$ then $\hat{\theta_{t+1}}\left(\hat{\theta_{0}}, \hat{\gamma_{0}}\right)$ has the following limit representation ${ }^{12}$ :

10 This can be done by considering two cases, namely $\hat{\varphi}_{0} \geq 0$ and $\hat{\varphi}_{0} \leq 0$. The former yields $y_{\tau} \stackrel{\tau \rightarrow \infty}{\Rightarrow} 0$ while the later does $v K_{\tau+1} y_{\tau+1} \stackrel{\tau \rightarrow \infty}{\Rightarrow} \hat{\varphi}_{0}$. The final result then is straightforward.

11 The expression $\hat{\theta_{1}}\left(\bar{\theta}, \hat{\gamma}_{0}\right)$ is defined only if $\hat{\varphi}_{0} \geq 0$, i.e., $\hat{\gamma}_{0} \leq(v-1) \hat{\theta_{0}}$.

12 If $\hat{\gamma}_{\tau}\left(\hat{\theta_{0}}, \hat{\gamma_{0}}\right)=0, \hat{\gamma}_{\tau}\left(\hat{\theta_{0}}, \hat{\gamma_{0}}\right)=\nu \eta\left(\hat{\theta_{\tau}}\left(\hat{\theta_{0}}, \hat{\gamma_{0}}\right), \bar{\theta}\right)-\hat{\theta_{\tau}}\left(\hat{\theta_{0}}, \hat{\gamma_{0}}\right)$ or $\hat{\theta_{\tau}}\left(\hat{\theta_{0}}, \hat{\gamma_{0}}\right)=\bar{\theta}$ for some $\tau$ the function $\hat{\theta_{\tau+1}}\left(\hat{\theta_{0}}, \hat{\gamma}_{0}\right)$ is not a limit function any more, but after all derivations have been made those functions will never be evaluated at such points. 


$$
\hat{\theta_{t+1}}\left(\hat{\theta_{0}}, \hat{\gamma_{0}}\right)=\lim _{\tau \rightarrow \infty} \theta_{\tau+t+1}\left(\theta_{\tau+t}\left(\ldots\left(\hat{\theta_{0}}, \hat{\gamma_{0}}\right)\right), \gamma_{\tau+t}\left(\ldots\left(\hat{\theta_{0}}, \hat{\gamma_{0}}\right)\right)\right) .
$$

The main use of that trick is to substitute complex functions $\theta_{\tau}\left(\theta_{\tau-1}\left(\ldots\left(\theta_{\tau-t}, \gamma_{\tau-t}\right)\right), \gamma_{t-1}\left(\ldots\left(\theta_{\tau-t}, \gamma_{\tau-t}\right)\right)\right)$ by theirs limit analogs for very large $t$ when the measure of "low quality goods" becomes negligible compare to the measure of "high quality goods". Limit functions $\hat{\theta_{t}}\left(\hat{\theta_{0}}, \hat{\gamma_{0}}\right)$ would have been exactly the same as $\theta_{\tau}\left(\theta_{\tau-1}\left(\ldots\left(\theta_{\tau-t}, \gamma_{\tau-t}\right)\right), \gamma_{t-1}\left(\ldots\left(\theta_{\tau-t}, \gamma_{\tau-t}\right)\right)\right)$ if there had been no entry of new sellers ${ }^{13}$.

Now let us fix $\hat{\theta_{0}}=\theta^{(k)}$ and take any $\hat{\gamma_{0}} \in\left(0, \frac{v-1}{\delta} \theta^{(k)}\right)$. If for some $\tau \geq 0$ we have obtained the functions $\hat{\theta_{\tau}}\left(\hat{\theta_{0}}, \hat{\gamma_{0}}\right)=\hat{\theta_{\tau}}\left(\hat{\gamma_{0}}\right)$ and $\hat{\gamma_{\tau}}\left(\hat{\theta_{0}}, \hat{\gamma_{0}}\right)=\hat{\gamma_{\tau}}\left(\hat{\gamma_{0}}\right)$, and at the same time $0<\hat{\gamma}_{\tau} \leq \eta \eta\left(\hat{\theta_{\tau}}, \bar{\theta}\right)-\hat{\theta_{\tau}}$ then there exists the next function, namely $\hat{\theta_{\tau+1}}\left(\hat{\gamma}_{0}\right)=\hat{\theta_{1}}\left(\hat{\theta_{\tau}}\left(\hat{\gamma}_{0}\right), \hat{\gamma_{\tau}}\left(\hat{\gamma}_{0}\right)\right)$ such that $\hat{\theta_{\tau+1}}\left(\hat{\gamma}_{0}\right) \in\left[\hat{\theta_{\tau}}, \bar{\theta}\right]$ for all $\hat{\gamma}_{0} \in\left(0, \frac{v-1}{\delta} \theta^{(k)}\right)$.

We will show that $\exists \hat{t} \geq 0$ and $\exists \hat{\gamma} \in\left(0, \frac{v-1}{\delta} \theta^{(k)}\right)$ such that either $\hat{\gamma}_{\hat{t}}(\hat{\gamma})=0$, or $\hat{\gamma}_{\hat{t}}(\hat{\gamma})>v \eta\left(\hat{\theta_{\hat{t}}}(\hat{\gamma}), \bar{\theta}\right)-\hat{\theta_{\hat{t}}}(\hat{\gamma})$.

Suppose not, that means that for any $t \geq 0$ and any $\hat{\gamma}_{0} \in\left(0, \frac{v-1}{\delta} \theta^{(k)}\right) \exists \hat{\theta}_{t}\left(\hat{\gamma}_{0}\right)$ and $\exists \hat{\gamma}_{t}\left(\hat{\gamma}_{0}\right)$ such that $0<\hat{\gamma}_{t} \leq \nu \eta\left(\hat{\theta_{t}}, \bar{\theta}\right)-\hat{\theta_{t}}$ and $\theta^{(k)} \leq \hat{\theta_{t}} \leq \bar{\theta}$. Let fix any $\hat{\gamma} \in\left(0, \frac{v-1}{\delta} \theta^{(k)}\right)$ and get infinite sequences $\left\{\hat{\theta}_{t}(\hat{\gamma})\right\}_{t=0}^{\infty}$ and $\left\{\hat{\gamma}_{t}(\hat{\gamma})\right\}_{t=0}^{\infty}$. The former is weakly increasing and bounded so $\exists \lim _{t \rightarrow \infty} \hat{\theta_{t}}=\hat{\theta_{\infty}} \leq \bar{\theta}$. But this implies that the later has a limit either $\lim _{t \rightarrow \infty} \hat{\gamma_{t}}=\lim _{t \rightarrow \infty}\left\{\nu \eta\left(\hat{\theta_{t}}, \hat{\theta_{t+1}}\right)-\hat{\theta_{t}}\right\}=v \eta\left(\hat{\theta_{\infty}}, \hat{\theta_{\infty}}\right)-\hat{\theta_{\infty}}=(v-1) \hat{\theta_{\infty}}>0$. Taking a limit of the indifference equation $\delta \hat{\gamma}_{t+1}=\hat{\gamma}_{t}-\left(\hat{\theta_{t+1}}-\hat{\theta_{t}}\right)$ gives rise to a contradiction:

$$
\delta(v-1) \hat{\theta}_{\infty}=\lim _{t \rightarrow \infty} \delta \hat{\gamma}_{t+1}=\lim _{t \rightarrow \infty}\left\{\hat{\gamma}_{t}-\left(\hat{\theta}_{t+1}-\hat{\theta_{t}}\right)\right\}=\lim _{t \rightarrow \infty} \hat{\gamma}_{t}=(v-1) \hat{\theta}_{\infty} .
$$

So, only two possibilities are left:

a) Case 1. $\exists \hat{t}$ and $\exists \hat{\gamma} \in\left(0, \frac{v-1}{\delta} \theta^{(k)}\right)$ such that for all $t=1, \ldots, \hat{t}-1$ and all $\hat{\gamma}_{0} \in(0, \hat{\gamma})$ $\hat{\gamma}_{t}>0, m\left(\hat{\theta_{t-1}}, \bar{\theta}\right) \geq \hat{\theta_{t-1}}+\hat{\gamma}_{t-1}, \hat{\gamma}_{\hat{t}}>0$ while $\eta \eta\left(\hat{\theta_{t-1}}(\hat{\gamma}), \bar{\theta}\right)<\hat{\theta_{\hat{t}-1}}(\hat{\gamma})+\hat{\gamma}_{\hat{t}-1}(\hat{\gamma})$; and

\footnotetext{
13 No entry case is described in Janssen and Roy (1999a).
} 
b) Case 2. $\exists \hat{t}$ and $\exists \hat{\gamma} \in\left(0, \frac{v-1}{\delta} \theta^{(k)}\right)$ such that for all $t=1, \ldots, \hat{t}$ and all $\hat{\gamma}_{0} \in(0, \hat{\gamma})$ $\hat{\gamma}_{t}>0$ and $\nu \eta\left(\hat{\theta_{t-1}}, \bar{\theta}\right) \geq \hat{\theta}_{t-1}+\hat{\gamma}_{t-1}$ while $\hat{\gamma}_{\hat{t}}(\hat{\gamma})=0$.

The detailed proof of the Cases is on request. We prove that in the Case 1 $\exists \overline{\varepsilon_{S}}>0$ such that for any $T \exists \bar{t}>T, \exists \tilde{\theta_{1}}(\bar{t}) \in\left(\theta_{S}-\varepsilon_{\mu}, \theta_{S}\right)$ and $\exists \Theta_{\bar{t}}\left(\tilde{\theta_{1}}\right)$ such that $\theta_{\bar{t}}\left(\tilde{\theta_{1}}\right)=\bar{\theta}$ and $s_{\bar{t}}\left(\tilde{\theta_{1}}\right)>\overline{\varepsilon_{S}}$. In other words in this case there exist infinite number equilibrium sequences such that all goods are traded in the last period.

In the Case 2 we define $\theta^{(k+1)}$ as $\theta^{(k+1)}=\hat{\theta_{\hat{t}}}(\hat{\gamma})$. We show that $\theta^{(k+1)} \in\left(v \theta^{(k)}, \bar{\theta}\right]$. Then we prove that either we have the same result as in the Case 1 , or for any $\overline{\varepsilon_{S}}>0$ and $\quad \overline{\varepsilon_{\theta}}>0 \quad \exists T_{S}^{(k+1)}>T_{S}^{(k)} \quad$ such that for all $t>T_{S}^{(k+1)}$ $\exists U_{t}^{(k+1)}=\left(\underline{\theta}_{1}(t, k+1), \bar{\theta}_{1}(t, k+1)\right) \subset U_{t}^{(k)}$ and $\exists \Theta_{t}\left(U_{t}^{(k+1)}\right)$ such that for all $\theta_{1} \in U_{t}^{(k+1)}$ $\left|\theta_{t}\left(\theta_{1}\right)-\theta^{(k+1)}\right|<\overline{\varepsilon_{\theta}}, 0 \leq s_{t}\left(\underline{\theta}_{1}\right)<\overline{\varepsilon_{S}}$ and $s_{t}\left(\bar{\theta}_{1}\right)>(v-1) \theta_{t}\left(\bar{\theta}_{1}\right)-\overline{\varepsilon_{s}}$.

\section{Proof of Theorem 4.1.}

Consequently applying Propositions 4.1 and 4.2 we get the following result: for any $\varepsilon_{S}>0$ and $\varepsilon_{\theta}>0 \quad \exists T_{S}$ such that for all $t \geq T_{S} \exists U_{t}^{S}=\left(\theta_{1}^{S}(t), \theta_{S}\right)$ and $\exists \Theta_{t}\left(U_{t}^{S}\right)$ such that for any $\theta_{1} \in U_{t}^{S} \theta_{t}\left(\theta_{1}\right) \in\left(\theta_{S}, \theta_{S}+\varepsilon_{\theta}\right)$ and $s_{t}\left(\theta_{1}^{S}\right)>(v-1) \theta_{t}\left(\theta_{1}^{S}\right)-\varepsilon_{S}$. Now we can see that we are under the conditions of Proposition 4.3 if we take $\theta^{(1)}=\theta_{S}<\bar{\theta}$. Here we distinguish three cases.

a) Case 1. For any $k=1, \ldots \infty$ there exists $\theta^{(k+1)} \in\left(\nu \theta^{(k)}, \bar{\theta}\right)$ such that for any $\overline{\varepsilon_{S}}>0$ and $\quad \overline{\varepsilon_{\theta}}>0 \quad \exists T_{S}^{(k+1)} \quad$ such that for all $t>T_{S}^{(k+1)}$ $\exists U_{t}^{(k+1)}=\left[\underline{\theta}_{1}(t, k+1), \bar{\theta}_{1}(t, k+1)\right] \subset U_{t}^{(k)}$ and $\exists \Theta_{t}\left(U_{t}^{(k+1)}\right)$ such that:

- for all $\theta_{1} \in U_{t}^{(k+1)} \theta_{t}\left(\theta_{1}\right)-\theta^{(k+1)} \mid<\overline{\varepsilon_{\theta}}$;

- $\quad 0 \leq s_{t}\left(\underline{\theta}_{1}(t, k+1)\right)<\overline{\varepsilon_{S}}$;

- $s_{t}\left(\bar{\theta}_{1}(t, k+1)\right)>(v-1) \theta_{t}\left(\bar{\theta}_{1}(t, k+1)\right)-\overline{\varepsilon_{S}}$. 
But in this case we get infinite sequence $\left\{\theta^{(k)}\right\}_{k=1}^{\infty}$ where $\theta^{(k+1)}>v \theta^{(k)}$, that contradicts with $\theta^{(k)}<\bar{\theta}$ as $\lim _{k \rightarrow \infty} \theta^{(k)}=+\infty$. So after some steps $\hat{k}$ we must meet either the Case 2 or Case 3.

b) Case 2. There exists $\theta^{(\hat{k})}$ such that there does not exist $\hat{\theta}^{(k+1)} \in\left(\nu \hat{\theta}^{(k)}, \bar{\theta}\right]$. In accordance with Proposition 4.3 we can make a conclusion: $\exists \overline{\varepsilon_{S}}>0$ such that for any $T \exists \bar{t}>T, \exists \tilde{\theta_{1}}(\bar{t})$ and $\exists \Theta_{\bar{t}}\left(\tilde{\theta_{1}}\right)$ such that $\theta_{\bar{t}}\left(\tilde{\theta_{1}}\right)=\bar{\theta}$ and $s_{\bar{t}}\left(\tilde{\theta_{1}}\right)>\overline{\varepsilon_{S}}$. In other words there are infinite number of equilibrium sequences such that all goods are sold in the last period and the last marginal surplus is strictly positive and separated from zero, $s_{\bar{t}}\left(\tilde{\theta_{1}}\right)>\overline{\varepsilon_{S}}>0$.

In this case we can construct infinite number of dynamic equilibria by concatenating equilibrium sequences, e.g. we take $\Theta_{t}=\left\{\theta_{\tau}\right\}_{\tau=1}^{t}$ and let a dynamic equilibrium be the following sequence of marginal sellers: $\left\{\bar{\theta}_{\tau}\right\}_{\tau=1}^{\infty}$ such that $\bar{\theta}_{\tau}=\theta_{\tau}$ if $\tau \leq t$ and $\bar{\theta}_{\tau}=\theta_{\tau-t}$ if $\tau>t$.

c) Case 3. There exists $\theta^{(\hat{k})}$ such that there exists $\hat{\theta}^{(k+1)}=\bar{\theta}$.

Note here that $\hat{\theta}^{(k+1)}$ is determined in terms of the previous point $\theta^{(\hat{k})}$, the measure function $\mu(\theta)$ and parameters $\underline{\theta}, v$ and $\delta$. In other words $\hat{\theta}^{(k+1)}=\Omega\left(\hat{\theta}^{(k)}, \mu(\theta), \underline{\theta}, v, \delta\right)$ where $\Omega\left(\hat{\theta}^{(k)}, \mu(\theta), \underline{\theta}, v, \delta\right)$ is some operator. Therefore the case when $\Omega\left(\hat{\theta}^{(k)}, \mu(\theta), \underline{\theta}, v, \delta\right)=\bar{\theta}$ is non-generic.

\section{Proof of Proposition 4.4.}

We begin with solving the system of indifference equations (4) w.r.t. $p_{\tau}$ :

$$
p_{\tau}-\theta_{\tau}\left(1-\delta^{t-\tau}\right)=\frac{p_{1}-\theta_{1}\left(1-\delta^{t-1}\right)}{\delta^{\tau-1}}, \tau=1, \ldots, t-1 .
$$


We will look for such a sequence of functions $\left\{\theta_{\tau}\left(\theta_{1}\right)\right\}_{\tau=1}^{t}$ satisfying (4) that $\theta_{\tau+1}<\theta_{\tau}$ for all $\tau=1, \ldots, t-2$. In this case we have $p_{\tau}\left(\theta_{\tau}\right)=\frac{v}{\mu\left(\underline{\theta}, \theta_{\tau}\right)} \int_{\underline{\theta}}^{\theta_{\tau}} \theta d \mu$, and $\frac{d p_{\tau}}{d \theta_{\tau}}\left(\theta_{S}\right)=a$. Substituting this into the first differential of (23) we get $\frac{d \theta_{\tau}}{d \theta_{1}}\left(\theta_{S}\right)=\frac{1}{\delta^{\tau-1}} \frac{\left(1-a-\delta^{t-1}\right)}{\left(1-a-\delta^{t-\tau}\right)}$.

Hence, we can write $\theta_{\tau}\left(\theta_{1}\right)$ and $s_{\tau}\left(\theta_{1}\right)$ as $\theta_{\tau}\left(\theta_{1}\right)=\theta_{S}-\frac{d \theta_{\tau}}{d \theta_{1}}\left(\theta_{S}\right)\left(\theta_{S}-\theta_{1}\right)+o\left(\theta_{1}-\theta_{S}\right)$ and $s_{\tau}\left(\theta_{1}\right)=-(1-a) \frac{d \theta_{\tau}}{d \theta_{1}}\left(\theta_{S}\right)\left(\theta_{1}-\theta_{S}\right)+o\left(\theta_{1}-\theta_{S}\right)$. Then

$$
\begin{aligned}
\theta_{\tau+1}-\theta_{\tau} & =\left(-\frac{1}{\delta^{\tau}} \frac{\left(1-a-\delta^{t-1}\right)}{\left(1-a-\delta^{t-\tau-1}\right)}+\frac{1}{\delta^{\tau-1}} \frac{\left(1-a-\delta^{t-1}\right)}{\left(1-a-\delta^{t-\tau}\right)}\right)\left(\theta_{S}-\theta_{1}\right)+o\left(\theta_{1}-\theta_{S}\right)= \\
& =-\frac{\left(1-a-\delta^{t-1}\right)(1-\delta)(1-a)}{\delta^{\tau}\left(1-a-\delta^{t-\tau}\right)\left(1-a-\delta^{t-\tau-1}\right)}\left(\theta_{S}-\theta_{1}\right)+o\left(\theta_{1}-\theta_{S}\right) .
\end{aligned}
$$

It follows that if $a<1-\delta$ then $\frac{d \theta_{\tau}}{d \theta_{1}}\left(\theta_{S}\right)>1$. Thus there exists a neighborhood, namely $U_{t-1}^{0}=\left(\theta_{1}^{0}(t-1), \theta_{S}\right)$, such that for all $\theta_{1} \in U_{t-1}^{0} \theta_{\tau}<\theta_{S}$ and, therefore, $\theta_{\tau+1}<\theta_{\tau}$, and $s_{\tau}>0$. Therefore there exists a sequence of functions $\left\{\theta_{\tau}\left(\theta_{1}\right)\right\}_{\tau=1}^{t-1}$ such that all conditions to be proved are satisfied except the last one and we only have to show that if $a \in(0, \tilde{a}(\delta))$ then there exists $\theta_{t}\left(\theta_{1}\right)$ and $U_{t}^{0}=\left(\theta_{1}^{0}(t), \theta_{S}\right) \in U_{t-1}^{0}$ such that for all $\theta_{1} \in U_{t}^{0} \quad 0<\theta_{t}\left(\theta_{1}\right)-\theta_{S}<\varepsilon_{\gamma} \frac{1}{\delta} s_{t}\left(\theta_{1}\right)$.

Given the structure of $\left\{\theta_{\tau}\left(\theta_{1}\right)\right\}_{\tau=1}^{t}$ we can write:

$$
p_{t}\left(\theta_{1}\right)=p_{t}\left(\theta_{1}\left(\theta_{1}\right), \theta_{2}\left(\theta_{1}\right), \ldots, \theta_{t}\left(\theta_{1}\right)\right)=v \frac{t \underline{\theta}_{\underline{\theta}}^{\theta_{t}\left(\theta_{1}\right)} \theta d \mu-\sum_{\tau=1}^{t-1} \int_{\underline{\theta}}^{\theta_{\tau}\left(\theta_{1}\right)} \theta d \mu}{t \mu\left(\underline{\theta}, \theta_{t}\left(\theta_{1}\right)\right)-\sum_{\tau=1}^{t-1} \mu\left(\underline{\theta}, \theta_{\tau}\left(\theta_{1}\right)\right)},
$$

and, consequently,

$$
\begin{aligned}
d_{S} p_{t} & =a\left(t d_{S} \theta_{t}-\sum_{\tau=1}^{t-1} d_{S} \theta_{\tau}\right)=a\left(t d_{S} \theta_{t}-d_{S} \theta_{1} \sum_{\tau=1}^{t-1} \frac{1}{\delta^{\tau-1}} \frac{\left(1-a-\delta^{t-1}\right)}{\left(1-a-\delta^{t-\tau}\right)}\right)= \\
& =a\left(t d_{S} \theta_{t}-d_{S} \theta_{1} \frac{1-a-\delta^{t-1}}{\delta^{t-1}} \sum_{\tau=1}^{t-1} \frac{\delta^{\tau}}{1-a-\delta^{\tau}}\right)
\end{aligned}
$$

Substituting this into the first differential of (4) yields 


$$
\frac{d \theta_{t}}{d \theta_{1}}\left(\theta_{S}\right)=\frac{1-a-\delta^{t-1}}{a t \delta^{t-1}}\left(a \sum_{\tau=1}^{t-1} \frac{\delta^{\tau}}{1-a-\delta^{\tau}}-1\right)
$$

and, therefore,

$$
\theta_{t}=\theta_{S}+\frac{d \theta_{t}}{d \theta_{1}}\left(\theta_{S}\right)\left(\theta_{1}-\theta_{S}\right)+o\left(\theta_{1}-\theta_{S}\right)
$$

This implies that there exists a neighborhood $\overline{U_{t}^{0}}=\left(\overline{\theta_{1}^{0}(t)}, \theta_{S}\right) \in U_{t-1}^{0}$, such that for all $\theta_{1} \in \overline{U_{t}^{0}} \quad \theta_{t}>\theta_{S}$ as long as $a \sum_{\tau=1}^{t-1} \frac{\delta^{\tau}}{1-a-\delta^{\tau}}<1$. So the condition $a \sum_{\tau=1}^{\infty} \frac{\delta^{\tau}}{1-a-\delta^{\tau}} \leq 1$ actually is a sufficient one. Note here that

$$
\begin{aligned}
& \sum_{\tau=1}^{\infty} \frac{a \delta^{\tau}}{1-a-\delta^{\tau}}<\sum_{\tau=1}^{\infty} \frac{a \delta^{\tau}}{1-a-\delta}=\frac{a \delta}{1-a-\delta} \sum_{\tau=1}^{\infty} \delta^{\tau-1}=\frac{a \delta}{(1-a-\delta)(1-\delta)}= \\
& =1+\frac{a-(1-\delta)^{2}}{(1-a-\delta)(1-\delta)},
\end{aligned}
$$

so if $a \leq(1-\delta)^{2}$ then $a \sum_{\tau=1}^{\infty} \frac{\delta^{\tau}}{1-a-\delta^{\tau}}<1$ and $a \leq(1-\delta)^{2}$ is a sufficient condition.

Then we check whether $s_{t}>0$ :

$$
d_{S} s_{t}=d_{S} p_{t}-d_{S} \theta_{t}=-\frac{1-a-\delta^{t-1}}{\delta^{t-1}}\left(1-\frac{1}{a t}\left(1-a \sum_{\tau=1}^{t-1} \frac{\delta^{\tau}}{1-a-\delta^{\tau}}\right)\right) d_{S} \theta_{1} .
$$

Hence, $\frac{d s_{t}}{d \theta_{1}}\left(\theta_{S}\right)<-\frac{1-a-\delta^{t-1}}{\delta^{t-1}}\left(1-\frac{1}{a t}\right)$ and it follows that $\frac{d s_{t}}{d \theta_{1}}\left(\theta_{S}\right)<0$ when $t>\frac{1}{a}$. So there exists $\overline{\overline{T_{0}}}=\left[\frac{1}{a}\right]+1$ such that for all $t>\overline{\overline{T_{0}}} \quad \exists \overline{\overline{U_{t}^{0}}}=\left(\overline{\overline{\theta_{1}^{0}(t)}}, \theta_{s}\right) \subset \overline{U_{t}^{0}}$ and $\exists \Theta^{2}{ }_{t}\left(\overline{\overline{U_{t}^{0}}}\right)$ such that for all $\theta_{1} \in \overline{\overline{U_{t}^{0}}} \theta_{t}\left(\theta_{1}\right)>\theta_{S}, s_{t}\left(\theta_{1}\right)>0 . \quad \theta_{\tau}\left(\theta_{S}\right)=\theta_{S}$ and $s_{\tau}\left(\theta_{S}\right)=0$ for all $\tau=1, \ldots, t$ by construction.

Finally we will prove that if $t$ is taken sufficiently large than $\exists \varepsilon_{\gamma}>0$ such that $0<\theta_{t}\left(\theta_{1}\right)-\theta_{S}<\varepsilon_{\gamma} \frac{1}{\delta} s_{t}\left(\theta_{1}\right)$. 
Let us consider the ratio $\frac{s_{t}\left(\theta_{1}\right)}{\theta_{t}\left(\theta_{1}\right)-\theta_{S}}$. It can be written as $\frac{s_{t}\left(\theta_{1}\right)}{\theta_{t}\left(\theta_{1}\right)-\theta_{S}}=\frac{\frac{d s_{t}}{d \theta_{1}}\left(\theta_{S}\right)\left(\theta_{1}-\theta_{S}\right)+o\left(\theta_{1}-\theta_{S}\right)}{\frac{d \theta_{t}}{d \theta_{1}}\left(\theta_{S}\right)\left(\theta_{1}-\theta_{S}\right)+o\left(\theta_{1}-\theta_{S}\right)}=\frac{a t+o\left(\left(\theta_{1}-\theta_{S}\right)^{0}\right)}{1-a \sum_{\tau=1}^{t-1} \frac{\delta^{\tau}}{1-a-\delta^{\tau}}}-1$. Therefore $\lim _{t \rightarrow \infty}\left[\lim _{\theta_{1} \rightarrow \theta_{S}-0} \frac{s_{t}\left(\theta_{1}\right)}{\theta_{t}\left(\theta_{1}\right)-\theta_{S}}\right]=\lim _{t \rightarrow \infty} \frac{a t}{1-a \sum_{\tau=1}^{t-1} \frac{\delta^{\tau}}{1-a-\delta^{\tau}}}-1=+\infty$.

This implies that we actually can take any $\varepsilon_{\gamma}>0$ such that $\exists T_{0}>\overline{\overline{T_{0}}}$ such that for all $t>T_{0} \exists U_{t}^{0}=\left(\theta_{1}^{0}(t), \theta_{S}\right)$ and $\exists \Theta^{2}{ }_{t}\left(U_{t}^{0}\right)$ such that $0<\theta_{t}\left(\theta_{1}\right)-\theta_{S}<\varepsilon_{\gamma} \frac{1}{\delta} s_{t}\left(\theta_{1}\right)$.

\section{Proof of Proposition 4.5.}

Suppose we have obtained an equilibrium sequence $\Theta_{k_{1}}\left(U_{k_{1}}^{0}\right)$, where $U_{k_{1}}^{0}=\left(\theta_{1}^{0}\left(k_{1}\right), \theta_{S}\right)$ such that for all $\tau=1, \ldots, k_{1} \quad \theta_{\tau}$ and $s_{\tau}$ can be represented as $\theta_{\tau}=\theta_{S}+\frac{d \theta_{\tau}}{d \theta_{1}}\left(\theta_{S}\right)\left(\theta_{1}-\theta_{S}\right)+o\left(\theta_{1}-\theta_{S}\right), \quad$ and $\quad s_{\tau}=\frac{d s_{\tau}}{d \theta_{1}}\left(\theta_{S}\right)\left(\theta_{1}-\theta_{S}\right)+o\left(\theta_{1}-\theta_{S}\right) \quad$ where $\frac{d s_{\tau}}{d \theta_{1}}\left(\theta_{S}\right)<0$ and $\frac{d \theta_{k_{1}}}{d \theta_{1}}\left(\theta_{S}\right)>0$, and for all $\theta_{1} \in U_{k_{1}}^{0} \theta_{\tau}\left(\theta_{1}\right)<\theta_{k_{1}}\left(\theta_{1}\right)<\theta_{S}$.

We introduce the following new variable:

$$
\alpha_{k_{1}} \equiv-\frac{d s_{k_{1}}}{d \theta_{k_{1}}}\left(\theta_{S}\right)=-\left(\frac{d \theta_{k_{1}}}{d \theta_{1}}\left(\theta_{S}\right)\right)^{-1} \frac{d s_{k_{1}}}{d \theta_{1}}\left(\theta_{S}\right)>0 \text {. }
$$

In terms of $\alpha_{k_{1}}$, surplus $s_{k_{1}}$ can be represented as $s_{k_{1}}\left(\theta_{1}\right)=\alpha_{k_{1}}\left(\theta_{k_{1}}-\theta_{S}\right)+o\left(\theta_{k_{1}}-\theta_{S}\right)$. There exists at least one of such a sequence, namely $\left\{\theta_{1}\right\}$, where $k_{1}=1$.

Now we will construct a new equilibrium sequence $\Theta_{t}\left(\Theta_{k_{1}}\right)$ in the following way. We will repeat the whole structure of $\Theta_{k_{1}} t$ times. In other words, for all $\tau=1, \ldots, t-1$ and for all $l=1, \ldots, k_{1}-1$ we put $\theta_{(\tau-1) k_{1}+l+1}\left(\theta_{1}\right)<\theta_{(\tau-1) k_{1}+l}\left(\theta_{1}\right)$ if $\theta_{l+1}\left(\theta_{1}\right)<\theta_{l}\left(\theta_{1}\right)$ and vice versa. Another rule is that for all $\tau=2, \ldots, t$ $\theta_{\tau k_{1}}\left(\theta_{1}\right)<\theta_{(\tau-1) k_{1}}\left(\theta_{1}\right)$. 
Having done this we can see that each of the sequences $\left\{\theta_{l}\left(\theta_{1}\right)\right\}_{l=(\tau-1) k_{1}+1}^{l=\tau k_{1}}$ for all $\tau=1, \ldots, t$ is an equilibrium sequence $\Theta_{k_{1}}$. Now we have to find $\theta_{t k_{1}+1}\left(\theta_{1}\right)$ such that for all $\tau=1, \ldots, t \quad \delta^{\tau k_{1}-1}\left(p_{\tau k_{1}}-\theta_{\tau k_{1}}\right)=\delta^{t k_{1}}\left(p_{t k_{1}+1}-\theta_{\tau k_{1}}\right)$, in other words, the seller of quality $\theta_{\tau k_{1}}$ must be indifferent between selling in time period $\tau k_{1}$ and $t k_{1}+1$. Loosely speaking we try to construct a sort of equilibrium sequence of type II using $\Theta_{k_{1}}$ as single components instead of $\theta_{\tau}$.

We will show that if $\alpha_{k_{1}}>\delta$ and $a k_{1} \sum_{\tau=1}^{\infty} \frac{\delta^{(\tau-1) k_{1}+1}}{\alpha_{k_{1}}-\delta^{(\tau-1) k_{1}+1}} \leq 1$ then it can be done for any $t$ and if $t>\frac{1-a}{a k_{1}}$ then $s_{t k_{1}+1}>0$. Applying the same procedure as in the proof of the Proposition 4.4 we get the following indifference equations:

$$
\delta^{(\tau-1) k_{1}}\left(p_{\tau k_{1}}-\left(1-\delta^{(t-\tau) k_{1}+1}\right) \partial_{\tau k_{1}}\right)=p_{k_{1}}-\left(1+\delta^{(t-1) k_{1}+1}\right)_{k_{1}} .
$$

Taking the first differentials of (25) at $\theta_{\tau}=\theta_{s}$ and using definition of $\alpha_{k_{1}}$ (24) we get:

$$
\delta^{(\tau-1) k_{1}}\left(-\alpha_{\tau k_{1}} d_{S} \theta_{\tau k_{1}}+\delta^{(t-\tau) k_{1}+1} d_{S} \theta_{\tau k_{1}}\right)=-\alpha_{k_{1}} d_{S} \theta_{k_{1}}+\delta^{(t-1) k_{1}+1} d_{S} \theta_{k_{1}} .
$$

Using the fact that $\alpha_{\tau k_{1}}=\alpha_{k_{1}}$ yields $\left(\alpha_{k_{1}}-\delta^{(t-\tau) k_{1}+1}\right) d_{S} \theta_{\tau k_{1}}=\frac{\alpha_{k_{1}}-\delta^{(t-1) k_{1}+1}}{\delta^{(\tau-1) k_{1}}} d_{S} \theta_{k_{1}}$, or $\frac{d \theta_{\tau k_{1}}}{d \theta_{k_{1}}}\left(\theta_{S}\right)=\frac{1}{\delta^{(\tau-1) k_{1}}} \frac{\alpha_{k_{1}}-\delta^{(t-1) k_{1}+1}}{\alpha_{k_{1}}-\delta^{(t-\tau) k_{1}+1}}$. Hence, we can write $\theta_{\tau k_{1}}\left(\theta_{1}\right)$ and $s_{\tau k_{1}}\left(\theta_{1}\right)$ as:

$$
\begin{aligned}
& \theta_{\tau k_{1}}\left(\theta_{1}\right)=\theta_{S}-\frac{d \theta_{\tau k_{1}}}{d \theta_{k_{1}}}\left(\theta_{S}\right) \cdot \frac{d \theta_{k_{1}}}{d \theta_{1}}\left(\theta_{S}\right)\left(\theta_{S}-\theta_{1}\right)+o\left(\theta_{1}-\theta_{S}\right), \text { and } \\
& s_{\tau k_{1}}\left(\theta_{1}\right)=\alpha_{\tau k_{1}}\left(\theta_{\tau k_{1}}-\theta_{S}\right)+o\left(\theta_{\tau k_{1}}-\theta_{S}\right)=\alpha_{k_{1}} \frac{d \theta_{\tau k_{1}}}{d \theta_{k_{1}}} \frac{d \theta_{k_{1}}}{d \theta_{1}}\left(\theta_{S}\right)\left(\theta_{1}-\theta_{S}\right)+o\left(\theta_{1}-\theta_{S}\right) . \text { Then } \\
& \theta_{(\tau+1) k_{1}}-\theta_{\tau k_{1}}=\left(-\frac{d \theta_{(\tau+1) k_{1}}}{d \theta_{k_{1}}}\left(\theta_{S}\right)+\frac{d \theta_{\tau k_{1}}}{d \theta_{k_{1}}}\left(\theta_{S}\right)\right) \frac{d \theta_{k_{1}}}{d \theta_{1}}\left(\theta_{S}\right)\left(\theta_{S}-\theta_{1}\right)+o\left(\theta_{1}-\theta_{S}\right)= \\
& =-\frac{\left(\alpha_{k_{1}}-\delta^{(t-1) k_{1}+1}\right)\left(1-\delta^{k_{1}}\right) \alpha_{k_{1}}}{\delta^{\tau k_{1}}\left(\alpha_{k_{1}}-\delta^{(t-\tau-1) k_{1}+1}\right)\left(\alpha_{k_{1}}-\delta^{(t-\tau) k_{1}+1}\right)} \frac{d \theta_{k_{1}}}{d \theta_{1}}\left(\theta_{S}\right)\left(\theta_{S}-\theta_{1}\right)+o\left(\theta_{1}-\theta_{S}\right)
\end{aligned}
$$


It follows that for any $\tau=1, \ldots, t \frac{d \theta_{\tau k_{1}}}{d \theta_{k_{1}}}\left(\theta_{S}\right) \geq 1$ as long as $\alpha_{k_{1}}>\delta^{14}$. Thus there exists a neighborhood, namely $\overline{U_{t k_{1}}^{0}}=\left(\overline{\theta_{1}^{0}\left(t k_{1}\right)}, \theta_{S}\right) \subset \overline{U_{k_{1}}^{0}}$, such that for all $\theta_{1} \in \overline{U_{t k_{1}}^{0}}$ $\theta_{\tau k_{1}}<\theta_{S}$ and, therefore, $\theta_{(\tau+1) k_{1}}<\theta_{\tau k_{1}}$, and $s_{\tau k_{1}}>0$. Therefore there exists a system of functions $\left\{\theta_{l}\left(\theta_{1}\right)\right\}_{l=1}^{l=t k_{1}}$ which satisfies all the indifference equations (25) such that all conditions of $\Theta_{t}\left(\Theta_{k_{1}}\right)$ are satisfied except the last one and now we have to find $\theta_{t k_{1}+1}\left(\theta_{1}\right) \geq \theta_{\tau k_{1}}\left(\theta_{1}\right)$ such that $s_{t k_{1}+1}\left(\theta_{1}\right)>0$.

Given the structure of $\left\{\theta_{l}\left(\theta_{1}\right)\right\}_{l=1}^{l=t k_{1}}$ we can write:

$$
p_{t k_{1}+1}\left(\theta_{k_{1}}\left(\theta_{1}\right), \ldots, \theta_{t k_{1}}\left(\theta_{1}\right), \theta_{t k_{1}+1}\left(\theta_{1}\right)\right)=v \frac{\left(t k_{1}+1\right) \int_{\underline{\theta}}^{\int_{t k_{1}+1}\left(\theta_{1}\right)} \theta d \mu-k_{1} \sum_{\tau=1}^{t} \int_{\underline{\theta}}^{\theta_{\tau k_{1}}\left(\theta_{1}\right)} \theta d \mu}{\left(t k_{1}+1\right) \mu\left(\underline{\theta}, \theta_{t k_{1}+1}\left(\theta_{1}\right)\right)-k_{1} \sum_{\tau=1}^{t} \mu\left(\underline{\theta}, \theta_{\tau k_{1}}\left(\theta_{1}\right)\right)},
$$

and, consequently,

$$
\begin{aligned}
d_{S} p_{t k_{1}+1} & =a\left(\left(t k_{1}+1\right) d_{S} \theta_{t k_{1}+1}-k_{1} \sum_{\tau=1}^{t} d_{S} \theta_{\tau k_{1}}\right)= \\
& =a\left(\left(t k_{1}+1\right) d_{S} \theta_{t k_{1}+1}-d_{S} \theta_{k_{1}} \frac{k_{1}\left(\alpha_{k_{1}}-\delta^{(t-1) k_{1}+1}\right)}{\delta^{t k_{1}}} \sum_{\tau=1}^{t} \frac{\delta^{(\tau-1) k_{1}+1}}{\alpha_{k_{1}}-\delta^{(\tau-1) k_{1}+1}}\right)
\end{aligned}
$$

Substituting this into the first differential of (25) yields

$$
\frac{d \theta_{t k_{1}+1}}{d \theta_{k_{1}}}\left(\theta_{S}\right)=\frac{\alpha_{k_{1}}-\delta^{(t-1) k_{1}+1}}{a\left(t k_{1}+1\right) \delta^{(t-1) k_{1}+1}}\left(a k_{1} \sum_{\tau=1}^{t} \frac{\delta^{(\tau-1) k_{1}+1}}{\alpha_{k_{1}}-\delta^{(\tau-1) k_{1}+1}}-1\right),
$$

and, therefore,

$$
\theta_{t k_{1}+1}=\theta_{S}+\frac{d \theta_{t k_{1}+1}}{d \theta_{k_{1}}}\left(\theta_{S}\right) \cdot \frac{d \theta_{k_{1}}}{d \theta_{1}}\left(\theta_{S}\right)\left(\theta_{1}-\theta_{S}\right)+o\left(\theta_{1}-\theta_{S}\right)
$$

This implies that there exists a neighborhood $\overline{U_{t k_{1}+1}^{0}}=\left(\overline{\theta_{1}^{0}\left(t k_{1}+1\right)}, \theta_{S}\right) \in \overline{U_{t k_{1}}^{0}}$, such that $\theta_{t k_{1}+1}>\theta_{S}$ as long as $\sum_{\tau=1}^{t} \frac{a k_{1} \delta^{(\tau-1) k_{1}+1}}{\alpha_{k_{1}}-\delta^{(\tau-1) k_{1}+1}}<1$. So the condition $\sum_{\tau=1}^{\infty} \frac{a k_{1} \delta^{(\tau-1) k_{1}+1}}{\alpha_{k_{1}}-\delta^{(\tau-1) k_{1}+1}} \leq 1$ actually is a sufficient one. Now we will check whether $s_{t k_{1}+1}>0$ :

\footnotetext{
14 Note that this condition is trivially satisfied for $t=1$.
} 


$$
d_{S} S_{t k_{1}+1}=-\frac{\alpha_{k_{1}}-\delta^{(t-1) k_{1}+1}}{\delta^{(t-1) k_{1}+1}}\left(\frac{1}{a\left(t k_{1}+1\right)}\left(a k_{1} \sum_{\tau=1}^{t} \frac{\delta^{(\tau-1) k_{1}+1}}{\alpha_{k_{1}}-\delta^{(\tau-1) k_{1}+1}}-1\right)+1\right) d_{S} \theta_{k_{1}},
$$

and, therefore,

$$
\frac{d s_{t k_{1}+1}}{d \theta_{1}}\left(\theta_{S}\right)<-\frac{\alpha_{k_{1}}-\delta^{(t-1) k_{1}+1}}{\delta^{(t-1) k_{1}+1}}\left(1-\frac{1}{a\left(t k_{1}+1\right)}\right) \frac{d \theta_{k_{1}}}{d \theta_{1}}\left(\theta_{S}\right) .
$$

It follows that $\frac{d s_{t k_{1}+1}}{d \theta_{1}}\left(\theta_{S}\right)<0$ when $t>\frac{1-a}{a k_{1}}$.

Now we will construct the desired equilibrium sequence $\Theta$ and our objective is to get $\Theta_{k_{m}}$ such that $a k_{m} \sum_{\tau=1}^{\infty} \frac{\delta^{(\tau-1) k_{m}+1}}{\alpha_{k_{m}}-\delta^{(\tau-1) k_{m}+1}} \leq 1$. We start from $k_{1}=1$ and $\Theta_{k_{1}}=\Theta_{1}$. Suppose that $\alpha_{k_{1}} \leq \delta$ and we cannot take $t>1$. In this case we take $t=1$, in other words we look for $\theta_{k_{1}+1}>\theta_{k_{1}}$. It follows that

$$
\frac{d \theta_{k_{1}+1}}{d \theta_{1}}\left(\theta_{S}\right)=\frac{a k_{1} \delta-\alpha_{k_{1}}+\delta}{a\left(k_{1}+1\right) \delta}=\frac{a k_{1} \delta+\left(\delta-\alpha_{k_{1}}\right)}{a\left(k_{1}+1\right) \delta}>0,
$$

and $\theta_{k_{1}+1}<\theta_{S}$, hence $s_{k_{1}+1}>0$, in some left neighborhood of $\theta_{S}$. We take $\Theta_{k_{2}} \equiv \Theta_{1}\left(\Theta_{k_{1}}\right)$, where $k_{2}=k_{1}+1$, as an initial equilibrium sequence and repeat the described procedure again.

Now we will prove that after some $n$ steps we get $\alpha_{k_{n}}>\delta$. To do so we take a lower limit of $\frac{\alpha_{k_{n+1}}}{\alpha_{k_{n}}}$ with $n \rightarrow \infty$. In this case $k_{n} \stackrel{n \rightarrow \infty}{\rightarrow} \infty$ as $k_{n+1}=k_{n}+1$ and we have:

$$
\begin{aligned}
\underset{n \rightarrow \infty}{\varliminf_{n \rightarrow+}} \frac{\alpha_{k_{n+1}}}{\alpha_{k_{n}}} & =\varliminf_{n \rightarrow \infty}\left(\frac{1}{\alpha_{k_{n}}} \frac{-\frac{d s_{k_{n}+1}}{d \theta_{k_{n}}}}{\frac{d \theta_{k_{n}+1}}{d \theta_{k_{n}}}}\right)=\varliminf_{n \rightarrow \infty}\left(-\frac{-\frac{\alpha_{k_{n}}-\delta}{\delta}\left(\frac{1}{a\left(k_{n}+1\right)}\left(\frac{\delta a k_{n}}{\alpha_{k_{n}}-\delta}-1\right)+1\right)}{\alpha_{k_{n}} \frac{a k_{n} \delta-\alpha_{k_{n}}+\delta}{a\left(k_{n}+1\right) \delta}}\right)= \\
& =\varliminf_{k_{n} \rightarrow \infty} \frac{\left(\delta-\alpha_{k_{n}}\right)(1-a)+a k_{n} \alpha_{k_{n}}}{\alpha_{k_{n}}\left(a k_{n} \delta-\alpha_{k_{n}}+\delta\right)}=\varliminf_{k_{n} \rightarrow \infty} \frac{1+\left(\frac{\delta}{\alpha_{k_{n}}}-1\right) \frac{1-a}{a k_{n}}}{\delta+\frac{\delta-\alpha_{k_{n}}}{a k_{n}}} .
\end{aligned}
$$

Suppose $\alpha_{k_{n}} \leq \delta$ for any $n$, then 


$$
\varliminf_{n \rightarrow \infty} \frac{\alpha_{k_{n+1}}}{\alpha_{k_{n}}}>\varliminf_{k_{n} \rightarrow \infty} \frac{1}{\delta+\frac{\delta}{a k_{n}}}=\frac{1}{\delta}>1
$$

But this implies that $\lim _{n \rightarrow \infty} \alpha_{k_{n}}=+\infty$ that contradicts with $\alpha_{k_{n}} \leq \delta$.

Hence, there exists some number $n$ such that we get $\Theta_{k_{n}}=\left\{\theta_{\tau}\left(\theta_{1}\right)\right\}_{\tau=1}^{\tau=k_{n}}$ and $\alpha_{k_{n}}>\delta$

Then we check whether $\sum_{\tau=1}^{\infty} \frac{a k_{n} \delta^{(\tau-1) k_{n}+1}}{\alpha_{k_{1}}-\delta^{(\tau-1) k_{n}+1}} \leq 1$ or not. If it is then we are done. In the case $\sum_{\tau=1}^{\infty} \frac{a k_{n} \delta^{(\tau-1) k_{n}+1}}{\alpha_{k_{1}}-\delta^{(\tau-1) k_{n}+1}}>1$ we will show that it is possible to find $\tau_{n}$ such that in equilibrium sequence $\Theta_{k_{n+1}}=\Theta_{\tau_{n}}\left(\Theta_{k_{n}}\right)$ we get $\theta_{\tau_{n} k_{n}+1}<\theta_{S}$.

We take $\tau_{n}=1$ and if $\frac{d \theta_{k_{n}+1}}{d \theta_{k_{n}}}\left(\theta_{S}\right)>0$ then $\Theta_{k_{n+1}}=\Theta_{1}\left(\Theta_{k_{n}}\right)$ and $\theta_{k_{n}+1}<\theta_{S}$ in some neighborhood of $\theta_{S}$. If $\frac{d \theta_{t k_{n}+1}}{d \theta_{k_{n}}}\left(\theta_{S}\right) \leq 0$ for some $t$, then it follows that $\frac{d \theta_{t k_{n}+1}}{d \theta_{k_{n}}}\left(\theta_{S}\right)=\frac{\alpha_{k_{n}}-\delta^{(t-1) k_{n}+1}}{a\left(t k_{n}+1\right) \delta^{(t-1) k_{n}+1}}\left(\sum_{\tau=1}^{t} \frac{a k_{n} \delta^{(\tau-1) k_{n}+1}}{\alpha_{k_{n}}-\delta^{(\tau-1) k_{n}+1}}-1\right)<0, \quad$ or $\quad \sum_{\tau=1}^{t} \frac{a k_{n} \delta^{(\tau-1) k_{n}+1}}{\alpha_{k_{n}}-\delta^{(\tau-1) k_{n}+1}}<1$.

On the other hand we have made an assumption that $\sum_{\tau=1}^{\infty} \frac{a k_{n} \delta^{(\tau-1) k_{n}+1}}{\alpha_{k_{n}}-\delta^{(\tau-1) k_{n}+1}}>1$. Therefore there exists $\tau_{n}$, such that $\sum_{j=1}^{\tau_{n}-1} \frac{a k_{n} \delta^{(j-1) k_{n}+1}}{\alpha_{k_{n}}-\delta^{(j-1) k_{n}+1}}<1$ while $\sum_{j=1}^{\tau_{n}} \frac{a k_{n} \delta^{(j-1) k_{n}+1}}{\alpha_{k_{n}}-\delta^{(j-1) k_{n}+1}} \geq 1$, and we have for $\Theta_{k_{n+1}}=\Theta_{\tau_{n}}\left(\Theta_{k_{n}}\right)$ :

$$
\begin{aligned}
& \frac{d \theta_{\tau_{n} k_{n}+1}}{d \theta_{k_{n}}}\left(\theta_{S}\right)=\frac{\alpha_{k_{n}}-\delta^{\left(\tau_{n}-1\right) k_{n}+1}}{a\left(\tau_{n} k_{n}+1\right) \delta^{\left(\tau_{n}-1\right) k_{n}+1}}\left(\sum_{j=1}^{\tau_{n}-1} \frac{a k_{n} \delta^{(j-1) k_{n}+1}}{\alpha_{k_{n}}-\delta^{(j-1) k_{n}+1}}-1\right)+\frac{a k_{n}}{a\left(\tau_{n} k_{n}+1\right)}= \\
& =\frac{1}{a\left(\tau_{n} k_{n}+1\right)}\left(\frac{\alpha_{k_{n}}-\delta^{\left(\tau_{n}-1\right) k_{n}+1}}{\delta^{\left(\tau_{n}-1\right) k_{n}+1}}\left(a k_{n} \sum_{j=1}^{\tau_{n}-1} \frac{a k_{n} \delta^{(j-1) k_{n}+1}}{\alpha_{k_{n}}-\delta^{(j-1) k_{n}+1}}-1\right)+a k_{n}\right)<\frac{a k_{n}}{a\left(\tau_{n} k_{n}+1\right)}<1 .
\end{aligned}
$$

In other words, $\theta_{k_{n}}<\theta_{k_{n+1}}=\theta_{\tau_{n} k_{n}+1}<\theta_{S}$ and $s_{k_{n+1}}=s_{\tau_{n} k_{n}+1}>0$, and we can continue with $\Theta_{k_{n+1}}=\Theta_{\tau_{n}}\left(\Theta_{k_{n}}\right)$. 
Now we will show that at some stage $m$ we will get $\sum_{\tau=1}^{\infty} \frac{a k_{m} \delta^{(\tau-1) k_{m}+1}}{\alpha_{k_{m}}-\delta^{(\tau-1) k_{m}+1}} \leq 1$. Suppose not, that means that for all $m \sum_{\tau=1}^{\infty} \frac{a k_{m} \delta^{(\tau-1) k_{m}+1}}{\alpha_{k_{m}}-\delta^{(\tau-1) k_{m}+1}}>1$. Then we can evaluate $\alpha_{k_{n+1}}=\alpha_{\tau_{n} k_{n}+1}$ taking into account that $\alpha_{k_{n}}>\delta$ :

$$
\begin{aligned}
\alpha_{k_{n+1}}=-\frac{\frac{d s_{\tau_{n} k_{n}+1}}{d \theta_{k_{m}}}\left(\theta_{S}\right)}{d s_{\tau_{n} k_{n}+1}}\left(\theta_{S}\right) & \frac{\frac{\alpha_{k_{n}}-\delta^{\left(\tau_{n}-1\right) k_{n}+1}}{d \theta_{k_{n}}}\left(\frac { 1 } { \delta ^ { ( \tau _ { n } - 1 ) k _ { n } + 1 } } \left(\frac{\alpha_{n}}{a\left(\tau_{n} k_{n}+1\right)}\left(\sum_{j=1}^{\tau_{n}} \frac{a k_{n} \delta^{(j-1) k_{n}+1}}{\alpha_{k_{n}}-\delta^{(j-1) k_{n}+1}}-1\right)+1\right.\right.}{\frac{\alpha_{k_{n}}-\delta^{\left(\tau_{n}-1\right) k_{n}+1}}{a\left(\tau_{n} k_{n}+1\right) \delta^{\left(\tau_{n}-1\right) k_{n}+1}}\left(\sum_{j=1}^{\tau_{n}} \frac{a k_{n} \delta^{(j-1) k_{n}+1}}{\alpha_{k_{n}}-\delta^{(j-1) k_{n}+1}}-1\right)}= \\
= & 1+\frac{a\left(\tau_{n} k_{n}+1\right)}{\sum_{j=1}^{\tau_{n}-1} \frac{a k_{n} \delta^{(j-1) k_{n}+1}}{\alpha_{k_{n}}-\delta^{(j-1) k_{n}+1}}-1+\frac{a k_{n} \delta^{\left(\tau_{n}-1\right) k_{n}+1}}{\alpha_{k_{n}}-\delta^{\left(\tau_{n}-1\right) k_{n}+1}}}>1+\frac{a \tau_{n} k_{n}}{\frac{a k_{n} \delta^{\left(\tau_{n}-1\right) k_{n}+1}}{\alpha_{k_{n}}-\delta^{\left(\tau_{n}-1\right) k_{n}+1}}}= \\
= & 1+\frac{\tau_{n}\left(\alpha_{k_{n}}-\delta^{\left(\tau_{n}-1\right) k_{n}+1}\right)}{\delta^{\left(\tau_{n}-1\right) k_{n}+1}}>1+\frac{\alpha_{k_{n}}-\delta}{\delta^{\left(\tau_{n}-1\right) k_{n}+1}}>1 .
\end{aligned}
$$

So, for all large $n \alpha_{k_{n}}>1>\delta$, and, therefore

$$
\begin{aligned}
\alpha_{k_{n+1}}= & 1+\frac{\tau_{n}\left(\alpha_{k_{n}}-\delta^{\left(\tau_{n}-1\right) k_{n}+1}\right)}{\delta^{\left(\tau_{n}-1\right) k_{n}+1}}>\frac{\tau_{n}\left(1-\delta^{\left(\tau_{n}-1\right) k_{n}+1}\right)+\delta^{\left(\tau_{n}-1\right) k_{n}+1}}{\delta^{\left(\tau_{n}-1\right) k_{n}+1}}> \\
& >\frac{\tau_{n}-\left(\tau_{n}-1\right) \delta^{\left(\tau_{n}-1\right) k_{n}+1}}{\delta^{\left(\tau_{n}-1\right) k_{n}+1}}=\frac{\left(\tau_{n}-1\right)-\left(\tau_{n}-1\right) \delta^{\left(\tau_{n}-1\right) k_{n}+1}+1}{\delta^{\left(\tau_{n}-1\right) k_{n}+1}}>\frac{1}{\delta^{\left(\tau_{n}-1\right) k_{n}+1}} .
\end{aligned}
$$

Then, it follows that $\lim \tau_{n} \neq 1$ because otherwise we would have $\tau_{n}=1$ for all large $n$ and, consequently,

$$
\varliminf_{n \rightarrow \infty} \frac{\alpha_{k_{n+1}}}{\alpha_{k_{n}}}=\varliminf_{k_{n} \rightarrow \infty} \frac{1+\left(\frac{\delta}{\alpha_{k_{n}}}-1\right) \frac{1-a}{a k_{n}}}{\delta+\frac{\delta-\alpha_{k_{n}}}{a k_{n}}}>\varliminf_{k_{n} \rightarrow \infty} \frac{1-\frac{1-a}{a k_{n}}}{\delta}=\frac{1}{\delta},
$$

and finally,

$$
\begin{gathered}
\varlimsup_{l \rightarrow \infty} \sum_{\tau=1}^{\infty} \frac{a k_{n+l} \delta^{(\tau-1) k_{n+l}+1}}{\alpha_{k_{n+l}}-\delta^{(\tau-1) k_{n+l}+1}} \leq \varlimsup_{l \rightarrow \infty} \sum_{\tau=1}^{\infty} \frac{a k_{n+l} \delta^{(\tau-1) k_{n+l}+1}}{\alpha_{k_{n+l}}-\delta}=\varlimsup_{l \rightarrow \infty} \frac{a k_{n+l} \delta}{\alpha_{k_{n+l}}-\delta} \sum_{\tau=1}^{\infty} \delta^{(\tau-1) k_{n+l}}= \\
=\varlimsup_{l \rightarrow \infty} \frac{a k_{n+l} \delta}{\left(\alpha_{k_{n+l}}-\delta\right)\left(1-\delta^{k_{n+l}}\right)}=\varlimsup_{l \rightarrow \infty} \frac{a k_{n+l} \delta}{\left(\alpha_{k_{n+l}}-\delta\right)}=\varlimsup_{l \rightarrow \infty} \frac{\delta a \frac{k_{n+l}}{\alpha_{k_{n+l}}}}{1-\frac{\delta}{\alpha_{k_{n+l}}}}=\delta a \varlimsup_{l \rightarrow \infty} \frac{k_{n+l}}{\alpha_{k_{n+l}}}=0,
\end{gathered}
$$


that contradicts with our assumption that for all $m \sum_{\tau=1}^{\infty} \frac{a k_{m} \delta^{(\tau-1) k_{m}+1}}{\alpha_{k_{m}}-\delta^{(\tau-1) k_{m}+1}}>1$.

So, $\lim _{n \rightarrow \infty} \tau_{n} \neq 1$ and there exists a subsequence $n_{l}$ such that $n_{l} \stackrel{l \rightarrow \infty}{\rightarrow \infty}$ and $\tau_{n_{l}} \geq 2$.

Taking an upper limit along that subsequence yields:

$$
\begin{aligned}
& \varlimsup_{l \rightarrow \infty}\left(\sum_{\tau=1}^{\infty} \frac{a k_{n_{l}+1} \delta^{(\tau-1) k_{n_{l}+1}+1}}{\alpha_{k_{n_{l}+1}}-\delta^{(\tau-1) k_{n_{l}+1}+1}}\right) \leq \varlimsup_{n_{l} \rightarrow \infty}\left(a k_{n_{l}+1} \delta \sum_{\tau=1}^{\infty} \frac{\delta^{(\tau-1) k_{n_{l}+1}}}{\alpha_{k_{n_{l}+1}}-\delta}\right)=\varlimsup_{n_{l} \rightarrow \infty}\left(\frac{a k_{n_{l}+1} \delta}{\alpha_{k_{n_{l}+1}}-\delta} \sum_{\tau=0}^{\infty} \delta^{\tau k_{n_{l}+1}}\right)= \\
& =\varlimsup_{n_{l} \rightarrow \infty}\left(\frac{a k_{n_{l}+1} \delta}{\alpha_{k_{n_{l}+1}}-\delta} \cdot \frac{1}{1-\delta^{k_{n_{l}+1}}}\right)=\varlimsup_{n_{l} \rightarrow \infty} \frac{a k_{n_{l}+1} \delta}{\alpha_{k_{n_{l}+1}}-\delta}=\varlimsup_{n_{l} \rightarrow \infty} \frac{a\left(\tau_{n_{l}} k_{n_{l}}+1\right) \delta}{\alpha_{k_{n_{l}+1}}-\delta} .
\end{aligned}
$$

Using the inequality (26) we get:

$$
\begin{aligned}
& \varlimsup_{l \rightarrow \infty}\left(a k_{n_{l}+1} \sum_{\tau=1}^{\infty} \frac{\delta^{(\tau-1) k_{n_{l}+1}+1}}{\alpha_{k_{n_{l}+1}}-\delta^{(\tau-1) k_{n_{l}+1}+1}}\right)=\varlimsup_{n_{l} \rightarrow \infty} \frac{a\left(\tau_{n_{l}} k_{n_{l}}+1\right) \delta}{\alpha_{k_{n_{l}+1}}-\delta} \leq \varlimsup_{n_{l} \rightarrow \infty} \frac{a\left(\tau_{n_{l}} k_{n_{l}}+1\right) \delta}{\frac{1}{\delta^{\left(\tau_{m_{l}}-1\right) k_{n_{l}}+1}}-\delta}= \\
& =\varlimsup_{n_{l} \rightarrow \infty} \frac{a\left(\tau_{n_{l}} k_{n_{l}}+1\right) \delta^{\left(\tau_{n_{l}}-1\right) k_{n_{l}}+2}}{1-\delta^{\left(\tau_{n_{l}}-1\right) k_{n_{l}}+2}}=a \delta^{2} \varlimsup_{\substack{n_{l} \rightarrow \infty \\
\tau_{n_{l}} \geq 2}}\left(\tau_{n_{l}} k_{n_{l}}+1\right) \delta^{\left(\tau_{n_{l}}-1\right) k_{n_{l}}}= \\
& =a \delta^{2} \varlimsup_{\substack{n_{l} \rightarrow \infty \\
\tau_{m_{l}} \geq 2}}\left(\left(\tau_{n_{l}}-1\right) k_{n_{l}}+1\right) \delta^{\left(\tau_{n_{l}}-1\right) k_{n_{l}}}+a \delta^{2} \varlimsup_{\substack{n_{l} \rightarrow \infty \\
\tau_{m_{l}} \geq 2}} k_{n_{l}} \delta^{\left(\tau_{n_{l}}-1\right) k_{n_{l}}} \leq \\
& \leq a \delta^{2} \varlimsup_{\left(\tau_{n_{l}}-1\right) k_{n_{l}} \rightarrow \infty}\left(\left(\tau_{n_{l}}-1\right) k_{n_{l}}+1\right) \delta^{\left(\tau_{n_{l}}-1\right) k_{n_{l}}}+a \delta^{2} \varlimsup_{k_{n_{l}} \rightarrow \infty} k_{n_{l}} \delta^{k_{n_{l}}}=a \delta^{2} \lim _{x \rightarrow \infty}(x+1) \delta^{x}=0 .
\end{aligned}
$$

Thus, we have proved the following statement: $\exists m$ and, therefore $\exists k_{m} \geq 1$, such that $a k_{m} \sum_{\tau=1}^{\infty} \frac{\delta^{(\tau-1) k_{m}+1}}{\alpha_{k_{m}}-\delta^{(\tau-1) k_{m}+1}} \leq 1$. But this implies that for any $t$ :

$$
\begin{aligned}
& \frac{d \theta_{t k_{m}+1}}{d \theta_{k_{m}}}\left(\theta_{S}\right)=\frac{\alpha_{k_{m}}-\delta^{(t-1) k_{m}+1}}{a\left(t k_{m}+1\right) \delta^{(t-1) k_{m}+1}}\left(a k_{m} \sum_{\tau=1}^{t} \frac{\delta^{(\tau-1) k_{m}+1}}{\alpha_{k_{m}}-\delta^{(\tau-1) k_{m}+1}}-1\right)<0, \text { and } \\
& \frac{d s_{t k_{m}+1}}{d \theta_{k_{m}}}\left(\theta_{S}\right)=<-\frac{\alpha_{k_{m}}-\delta^{(t-1) k_{m}+1}}{\delta^{(t-1) k_{m}+1}}\left(1-\frac{1}{a\left(t k_{m}+1\right)}\right)<0 \text { as long as } t>\frac{1-a}{a k_{m}} .
\end{aligned}
$$

So there exists $\overline{\overline{T_{0}}}=\left[\frac{1-a}{a k_{m}}\right]+1$ such that for all $t>\overline{\overline{T_{0}}}$ $\overline{\overline{U_{t k_{m}+1}^{0}}}=\left(\overline{\overline{\theta_{1}^{0}\left(t k_{m}+1\right)}}, \theta_{S}\right) \subset \overline{U_{k_{m}}^{0}}$ and $\exists \Theta_{t}\left(\overline{\overline{U_{t k_{m}+1}^{0}}}\right)$ such that for all $\theta_{1} \in \overline{\overline{U_{t k_{m}+1}^{0}}}$ 
$\theta_{t k_{m}+1}\left(\theta_{1}\right)>\theta_{S}, \quad s_{t k_{m}+1}\left(\theta_{1}\right)>0 . \quad \theta_{\tau}\left(\theta_{S}\right)=\theta_{S}$ and $s_{\tau}\left(\theta_{S}\right)=0$ for all $\tau=1, \ldots, t k_{m}+1$ by construction.

Finally we will prove that if $t$ is taken sufficiently large than $\exists \varepsilon_{\gamma}>0$ such that $\theta_{t k_{m}+1}\left(\theta_{1}\right)-\theta_{S}<\varepsilon_{\gamma} \frac{1}{\delta} s_{t k_{m}+1}\left(\theta_{1}\right)$. Let us consider the ratio $\frac{s_{t k_{m}+1}\left(\theta_{1}\right)}{\theta_{t k_{m}+1}\left(\theta_{1}\right)-\theta_{S}}$. It can be written as follows:

$$
\frac{s_{t k_{m}+1}\left(\theta_{1}\right)}{\theta_{t k_{m}+1}\left(\theta_{1}\right)-\theta_{S}}=\frac{\frac{d s_{t k_{m}+1}}{d \theta_{1}}\left(\theta_{S}\right)\left(\theta_{1}-\theta_{S}\right)+o\left(\theta_{1}-\theta_{S}\right)}{\frac{d \theta_{t k_{m}+1}}{d \theta_{1}}\left(\theta_{S}\right)\left(\theta_{1}-\theta_{S}\right)+o\left(\theta_{1}-\theta_{S}\right)}=\frac{a\left(t k_{m}+1\right)+o\left(\left(\theta_{1}-\theta_{S}\right)^{0}\right)}{1-a k_{m} \sum_{\tau=1}^{t} \frac{\delta^{(\tau-1) k_{m}+1}}{\alpha_{k_{m}}-\delta^{(\tau-1) k_{m}+1}}}-1 .
$$

Therefore

$$
\lim _{t \rightarrow \infty}\left[\lim _{\theta_{1} \rightarrow \theta_{S}-0} \frac{s_{t k_{m}+1}\left(\theta_{1}\right)}{\theta_{t k_{m}+1}\left(\theta_{1}\right)-\theta_{S}}\right]=\lim _{t \rightarrow \infty} \frac{a\left(t k_{m}+1\right)}{1-a k_{m} \sum_{\tau=1}^{t} \frac{\delta^{(\tau-1) k_{m}+1}}{\alpha_{k_{m}}-\delta^{(\tau-1) k_{m}+1}}}-1=+\infty .
$$

This implies that we actually can take any $\varepsilon_{\gamma}>0$ such that $\exists T_{0}>\overline{\overline{T_{0}}}$ such that for all $t>T_{0} \quad \exists U_{t k_{m}+1}^{0}=\left(\theta_{1}^{0}\left(t k_{m}+1\right), \theta_{S}\right)$ and $\exists \Theta_{t k_{m}+1}\left(U_{t k_{m}+1}^{0}\right)$ such that for all $\theta_{1} \in U_{t k_{m}+1}^{0}$ $0<\theta_{t k_{m}+1}-\theta_{S}<\varepsilon_{\gamma} \frac{1}{\delta} s_{t k_{m}+1}$. 